\title{
Complex Dimensions of Self-Similar Fractal Strings and Diophantine Approximation
}

\author{
Michel L. Lapidus and Machiel van Frankenhuysen
}

\section{CONTENTS}

1. Introduction

2. Dirichlet Polynomial Equations

3. Approximating a Nonlattice Equation by Lattice Equations

4. Complex Roots of a Nonlattice Dirichlet Polynomial

5. Self-Similar Fractal Strings

6. Explicit Formulas

7. Dimension-Free Regions

8. The Real Parts of the Complex Dimensions of a Nonlattice String

Acknowledgments

References

2000 AMS Subject Classification: Primary 11N05, 28A80, 58F03, 58F20; Secondary: 11M41, 58F11, 58F15, 58G25

Keywords: Dirichlet polynomial equations, complex roots and dimensions, Diophantine approximation, self-similar fractal strings
We study the solutions in $s$ of a "Dirichlet polynomial equation" $m_{1} r_{1}^{s}+\cdots+m_{M} r_{M}^{s}=1$. We distinguish two cases. In the lattice case, when $r_{j}=r^{k_{j}}$ are powers of a common base $r$, the equation corresponds to a polynomial equation, which is readily solved numerically by using a computer. In the nonlattice case, when some ratio $\log r_{j} / \log r_{1}, j \geq 2$, is irrational, we obtain information by approximating the equation by lattice equations of higher and higher degree. We show that the set of lattice equations is dense in the set of all equations, and deduce that the roots of a nonlattice Dirichlet polynomial equation have a quasiperiodic structure, which we study in detail both theoretically and numerically.

This question is connected with the study of the complex dimensions of self-similar strings. Our results suggest, in particular, that a nonlattice string possesses a set of complex dimensions with countably many real parts (fractal dimensions) which are dense in a connected interval. Moreover, we find dimension-free regions of nonlattice self-similar strings. We illustrate our theory with several examples.

In the long term, this work is aimed in part at developing a Diophantine approximation theory of (higher-dimensional) selfsimilar fractals, both qualitatively and quantitatively.

\section{INTRODUCTION}

Let $1>r_{1} \geq \cdots \geq r_{N}>0$ be $N$ positive real numbers. The equation

$$
r_{1}^{s}+\cdots+r_{N}^{s}=1
$$

has one real root, called $D$, and many complex roots. More generally, for $M$ scaling ratios $r_{0}>r_{1}>\cdots>$ $r_{M}>0$ (which we now assume to be unequal), and $\mathrm{mul}$ tiplicities $m_{j} \in \mathbb{C}(j=1, \ldots, M)$, a function of the form

$$
f(s)=m_{0} r_{0}^{s}+m_{1} r_{1}^{s}+\cdots+m_{M} r_{M}^{s}
$$

is a Dirichlet polynomial. In this paper, we study the complex solutions of the Dirichlet polynomial equation

$$
f(s)=0 \text {. }
$$


In Section 2, we introduce the lattice case, when $f(s)$ is a polynomial of $r^{s}$ for some $r>0$, and the nonlattice case, when $f$ cannot be so written. We recall some basic facts about the complex roots of Equation (1-3), and we prove a result about their density. In Section 3, we introduce an approximation procedure, allowing us to replace the study of a nonlattice equation by the study of a sequence of lattice equations. Thus, we find that the set of lattice equations is dense in the set of all Dirichlet polynomial equations (Section 2.3 and Section 3). It follows that the set of complex roots of a nonlattice equation has a quasiperiodic structure, in a very precise sense, amenable to computer experimentation (see, in particular, Theorem 3.6 and the comments following it). In Section 4, we implement this program. We discuss the structure of the complex roots of a nonlattice equation close to the line $\operatorname{Re} s=D$. The case $M=2$ is dealt with explicitly, using continued fractions. For $M>2$, we use a more implicit approach, based on a suitable Diophantine approximation. In this case, one could also use the LLL-algorithm [Lenstra et al. 82]. Our results are illustrated in a number of plots of the complex roots and density plots of their approximated real parts for several (generic and nongeneric) nonlattice equations.

Equation (1-1) - along with the more general Dirichlet polynomial equation (1-3), with $f$ given by (1-2)occurs often in many areas of mathematics. Next, we discuss one motivation, that of self-similar fractal strings, from the authors' recent book [Lapidus and van Frankenhuysen 00]. We note that in Section 6, we extend the discussion in order to deal with arbitrary "self-similar strings" (with scaling ratios of noninteger multiplicity). In that case, Equation (1-1) is replaced by an equation of the form (1-3).

A fractal string $\mathcal{L}$ (one-dimensional drum with fractal boundary, see [Lapidus 93, Lapidus and Pomerance 93, Lapidus and Maier 95] and [Lapidus and van Frankenhuysen 00, Chapters 1 and 2]) is a disjoint union of open intervals, the lengths of which form a sequence

$$
\mathcal{L}=l_{1}, l_{2}, l_{3}, \ldots
$$

of finite total length $|\mathcal{L}|=\sum_{j=1}^{\infty} l_{j}$. The geometric zeta function of $\mathcal{L}$ is defined as

$$
\zeta_{\mathcal{L}}(s)=\sum_{j=1}^{\infty} l_{j}^{s}
$$

which we assume to have a meromorphic continuation to the left of $D$, the abscissa of convergence of $\zeta_{\mathcal{L}}$. The geometric meaning of $D$ is that it coincides with the
Minkowski dimension of the boundary of $\mathcal{L}{ }^{1}$ The poles of $\zeta_{\mathcal{L}}$ (in a certain domain $W$; see Section 6 ) are called the complex dimensions of $\mathcal{L}$. In particular, $D$ is a complex dimension of $\mathcal{L}$.

A self-similar fractal string is defined by means of scaling ratios, see [Lapidus and van Frankenhuysen 00, Chapter 2] and Section 5. Let $N \geq 2$ and $r_{1}, \ldots, r_{N}$ be the scaling ratios of a self-similar fractal string $\mathcal{L}$. We always have $r_{j} \in(0,1)$ for $j=1, \ldots, N$ and in this setting, we need to assume that $r_{1}+\cdots+r_{N}<1$. The geometric zeta function of $\mathcal{L}$ is

$$
\zeta_{\mathcal{L}}(s)=\frac{1}{1-\sum_{j=1}^{N} r_{j}^{s}}
$$

(See Theorem 5.2.) Hence, the solutions to (1-1) are the complex dimensions of $\mathcal{L}$.

As an example of their geometric importance, all complex dimensions - and hence, for self-similar strings, all complex roots of (1-1) - enter into the explicit formula ${ }^{2}$ for the volume $V(\varepsilon)$ of the inner tubular neighborhood (of radius $\varepsilon$ ) of the boundary of $\mathcal{L}$ :

$$
V(\varepsilon)=\sum_{\omega} \frac{(2 \varepsilon)^{1-\omega}}{\omega(1-\omega)} \operatorname{res}\left(\zeta_{\mathcal{L}}(s) ; \omega\right)+2 \varepsilon \zeta_{\mathcal{L}}(0)+o(\varepsilon)
$$

where $\omega$ runs through the complex dimensions of $\mathcal{L}$. Since $\omega=D$ is a complex dimension, this formula expresses $V(\varepsilon)$ as a sum of the first term, ${ }^{3}$

$$
\frac{(2 \varepsilon)^{1-D}}{D(1-D)} \operatorname{res}\left(\zeta_{\mathcal{L}} ; D\right)
$$

of order $\varepsilon^{1-D}$, and the oscillatory terms $c_{\omega} \varepsilon^{1-\omega}$, for certain coefficients $c_{\omega}$. These terms have order $\varepsilon^{1-\operatorname{Re} \omega}$, and exhibit multiplicative oscillations of period $e^{2 \pi / \operatorname{Im} \omega}$.

A dimension-free region is a region in the complex plane such that its intersection with the set of complex dimensions is only $D$. If there exist positive numbers $C$ and $p$ such that

$$
\left\{\omega \in \mathbb{C}: \operatorname{Re} \omega \geq D-C(1+|\operatorname{Im} \omega|)^{-p}\right\}
$$

\footnotetext{
${ }^{1}$ Except in the trivial situation when $\mathcal{L}$ is a finite sequence, in which case the abscissa of convergence of $\zeta_{\mathcal{L}}$ is equal to $-\infty$ and $D=0$. (See Footnote 3 for the definition of the Minkowski dimension.)

${ }^{2}$ We assume here that $0 \in W$ (see Section 6 and Footnote 11) and that the complex dimensions $\omega$ are simple poles of $\zeta_{\mathcal{L}}$. See $(6-11)$ for the general formula. Throughout, $\operatorname{res}(g(s) ; \omega)$ denotes the residue of the meromorphic function $g=g(s)$ at $\omega$.

${ }^{3}$ Recall that the Minkowski (or box) dimension is defined as the unique value $D \in[0,1]$ for which $\lim _{\varepsilon \downarrow 0} V(\varepsilon) \varepsilon^{\delta-1}=0$ or $\infty$ according to whether $\delta>D$ or $\delta<D$; see, e.g., [Lapidus 93, Lapidus and Pomerance 93, Lapidus and Maier 95], [Lapidus and van Frankenhuysen 00, §1.1] and [Falconer 90, Chapter 3].
} 
is a dimension-free region, then (1-7) allows us to deduce, by techniques explained in [Lapidus and van Frankenhuysen 01a], that for every $\delta>0$,

$$
V(\varepsilon)=\mathcal{M}(D ; \mathcal{L}) \varepsilon^{1-D}\left(1+O(|\log \varepsilon|)^{\delta-(1 / p)}\right)
$$

as $\varepsilon \rightarrow 0^{+}$. Here, $\mathcal{M}(D ; \mathcal{L})=\frac{2^{1-D}}{D(1-D)} \operatorname{res}\left(\zeta_{\mathcal{L}} ; D\right)$ is the Minkowski content of the boundary of $\mathcal{L}$. It is defined by

$$
\mathcal{M}(D ; \mathcal{L})=\lim _{\varepsilon \downarrow 0} V(\varepsilon) \varepsilon^{-(1-D)},
$$

when this limit exists in $(0, \infty)$, in which case $\mathcal{L}$ is said to be Minkowski measurable; see, e.g., [Lapidus 93, Lapidus and Pomerance 93, Lapidus and Maier 95] or [Lapidus and van Frankenhuysen 00, §1.1]. In Section 7, we deduce from the results of Section 4 that nonlattice strings have a dimension-free region, which is of the form (1-9) when the nonlattice string is badly approximable by lattice strings. In general, the dimension-free region is much thinner, with a corresponding weaker form of (1-10).

The algorithms developed in Section 4 are used - along with our theoretical investigations - to approximate and plot the complex dimensions of a variety of nonlattice self-similar strings, as well as to better understand their rich structure, in terms of Diophantine properties of their scaling ratios (or weights); see, e.g., Examples 7.3-7.6 and Figures 8-12. Thus, the problem of finding the complex dimensions becomes accessible to numerical computation. We first solve this problem for lattice strings, in which case the associated equation is polynomial.

We end this paper with a theorem and several conjectures about the dimensions of fractality of a nonlattice string; see Section 8.

In closing this introduction, we note that although the theory of complex dimensions of self-similar sets has been developed so far mostly in the one-dimensional case in LLapidus and van Frankenhuysen 99, Lapidus and van Frankenhuysen 00] (i.e., in the case of self-similar strings), we expect that once it will have been suitably extended to the higher-dimensional case, the methods developed in the present paper will also apply to that more general situation.

Remark 1.1. A preliminary version of this paper was provided, in lesser generality, in the MSRI preprint [Lapidus and van Frankenhuysen 01b]. We note that, in particular, the class of Dirichlet polynomials of which we have studied the zeros in [Lapidus and van Frankenhuysen 01b] is significantly smaller than in the present paper. The increased generality allowed here is naturally motivated from a mathematical point of view since the corresponding equations arise frequently in the applications. Moreover, it enables us to study the complex dimensions of "generalized self-similar strings," as well as their dynamical counterpart for "self-similar flows." We refer to Section 6.3 for a discussion of self-similar flows and of their associated "dynamical zeta functions." In our theory, the study of the "prime orbit counting functions" of such flows leads to equations of the type (1-3). (See especially Remarks 6.5 and 6.4.) Analogously, general self-similar sets (with gaps) in $\mathbb{R}$ also lead to the study of these equations, as is explained in Section 5.

\section{DIRICHLET POLYNOMIAL EQUATIONS}

For an integer $M \geq 1$, let $r_{0}, \ldots, r_{M}$ be $M+1$ positive numbers such that $r_{0}>r_{1}>\cdots>r_{M}>0$ and let $m_{0}, \ldots, m_{M} \in \mathbb{C}$ be complex "multiplicities." Write

$$
w_{j}=-\log r_{j}
$$

for $j=0, \ldots, M$. In [Lapidus and van Frankenhuysen 01a], the numbers $w_{j}$ are interpreted as the weights of a self-similar flow associated with $\mathcal{L}$. Let

$$
f(s)=\sum_{j=0}^{M}-m_{j} r_{j}^{s}=\sum_{j=0}^{M}-m_{j} e^{-w_{j} s} .
$$

The $r_{j}$ are called the scaling ratios, and the $w_{j}$ are the weights. Without loss of generality, we assume the normalization $r_{0}=1$ and $m_{0}=-1$. Thus, $w_{0}=0$ and

$$
f(s)=1-\sum_{j=1}^{M} m_{j} r_{j}^{s}=1-\sum_{j=1}^{M} m_{j} e^{-w_{j} s} .
$$

\subsection{The Lattice and Nonlattice Case}

Consider the additive subgroup

$$
G=\mathbb{Z} w_{1}+\cdots+\mathbb{Z} w_{M}
$$

of the group of real numbers.

Definition 2.1. If $G$ is dense in $\mathbb{R}$, then $(2-3)$ is a nonlattice Dirichlet polynomial, and if $G$ is discrete in $\mathbb{R}$, then $(2-3)$ is a lattice Dirichlet polynomial.

In the lattice case, there exist a positive real number $w$ and natural numbers $k_{1}, \ldots, k_{M}$ without common factor such that $w_{j}=k_{j} w$ for $j=1, \ldots, M$. The number $r=e^{-w}$ is called the multiplicative generator of the function (2-3). Thus, $r_{j}=r^{k_{j}}$

for $j=1, \ldots, M$. 
Since $G$ is a free Abelian group, another way of phrasing this definition is as follows: The lattice case is when the rank of $G$ is one, and the nonlattice case is when this rank is $\geq 2$. We refine Definition 2.1 as follows: The generic nonlattice case is when the rank of $G$ is $M$.

Remark 2.2. The dichotomy lattice/nonlattice - also referred to as arithmetic/nonarithmetic in probability theory - comes from renewal theory (see [Feller 66, Chapter $\mathrm{X}]$ ) and was used in this and in a related context in, for example, [Lalley 89, Lalley 91], [Strichartz 90, Strichartz 93], [Lapidus 93, Kigami and Lapidus 93, Lapidus and van Frankenhuysen 99, Lapidus and van Frankenhuysen 01a] and the relevant references therein.

Remark 2.3. In a suitable sense, the nonlattice case is the generic case. (See [Lapidus and van Frankenhuysen $00, \S 2.3 .1]$ for a more precise discussion.) A key objective of the rest of this paper is to demonstrate the relationship between lattice and nonlattice equations as well as to understand the qualitative and quantitative differences between various nonlattice equations in terms of the Diophantine properties of their scaling ratios (or weights).

In view of our discussion in Section 1 and, in a more general setting in Section 5, it is then straightforward to apply our results about the complex roots of nonlattice equations to the complex dimensions of nonlattice selfsimilar strings.

\subsection{Examples of Dirichlet Polynomial Equations}

2.2.1 Linear Example. Take one scaling factor $r_{1}=$ $1 / 3$, with multiplicity $m_{1}=2$. The associated Dirichlet polynomial is

$$
f(s)=1-2 \cdot 3^{-s} .
$$

The complex roots are found by solving the equation

$$
2 \cdot 3^{-\omega}=1 \quad(\omega \in \mathbb{C}) .
$$

We find

$$
\mathcal{D}=\{D+i n \mathbf{p}: n \in \mathbb{Z}\},
$$

with $D=\log _{3} 2$ and $\mathbf{p}=2 \pi / \log 3$. (See Figure 1.) All complex roots are simple.

2.2.2 Quadratic Example. Take two scaling factors $r_{1}=1 / 2, r_{2}=1 / 4$, both with multiplicity $m_{1}=m_{2}=1$. The Dirichlet polynomial is

$$
f(s)=1-2^{-s}-4^{-s} .
$$

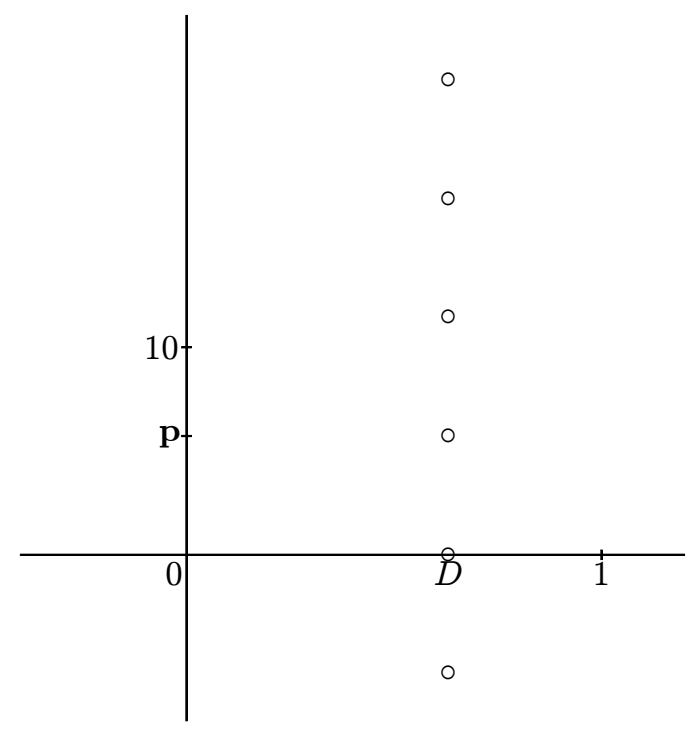

FIGURE 1. A diagram of the complex roots of a linear Dirichlet polynomial equation. $D=\log _{3} 2$ and $\mathbf{p}=$ $2 \pi / \log 3$.

The complex roots are found by solving the quadratic equation

$$
\left(2^{-\omega}\right)^{2}+2^{-\omega}=1 \quad(\omega \in \mathbb{C}) .
$$

We find the two solutions $2^{-\omega}=(-1+\sqrt{5}) / 2=\phi^{-1}$ and $2^{-\omega}=-\phi$, where

$$
\phi=\frac{1+\sqrt{5}}{2}
$$

is the golden ratio. Hence,

$$
\mathcal{D}=\{D+i n \mathbf{p}: n \in \mathbb{Z}\} \cup\{-D+i(n+1 / 2) \mathbf{p}: n \in \mathbb{Z}\},
$$

with $D=\log _{2} \phi$ and $\mathbf{p}=2 \pi / \log 2 . \quad$ (See Figure 2.) Again, all the roots are simple.

Remark 2.4. In the context of self-similar strings ([Lapidus and van Frankenhuysen 00, Chapter 2] and Section 5 below) Example 2.2.1 (respectively, 2.2.2) is related to the Cantor (respectively, Fibonacci) string; see [Lapidus and van Frankenhuysen 00, §2.2.1 and $\S 2.2 .2]$.

2.2.3 An Example with Multiple Roots. Figure 3 gives the complex roots of the Dirichlet polynomial

$$
f(s)=1-3 \cdot 9^{-s}-2 \cdot 27^{-s},
$$




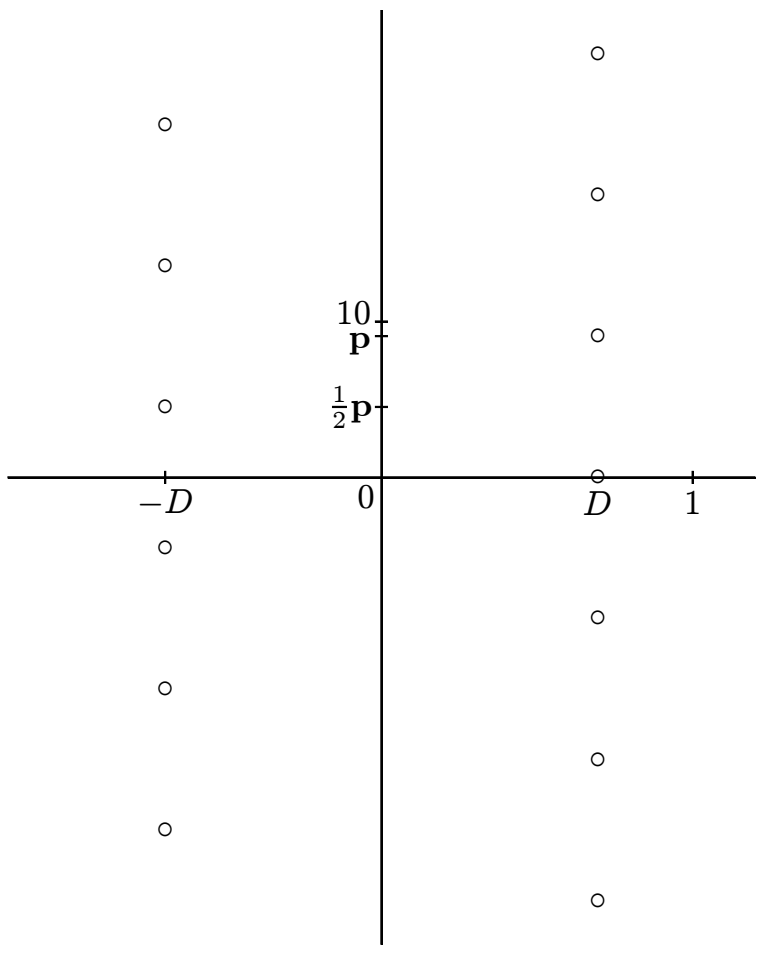

FIGURE 2. The complex roots of a quadratic Dirichlet polynomial equation. $D=\log _{2} \phi$ and $\mathbf{p}=2 \pi / \log 2$.

which factors as $(2 z-1)(z+1)^{2}=0$, with $z=3^{-s}$. Thus, we see that there is one line of simple roots $\omega=D+i n \mathbf{p}$ $\left(D=\log _{3} 2\right.$ and $\left.\mathbf{p}=2 \pi / \log 3, n \in \mathbb{Z}\right)$, corresponding to

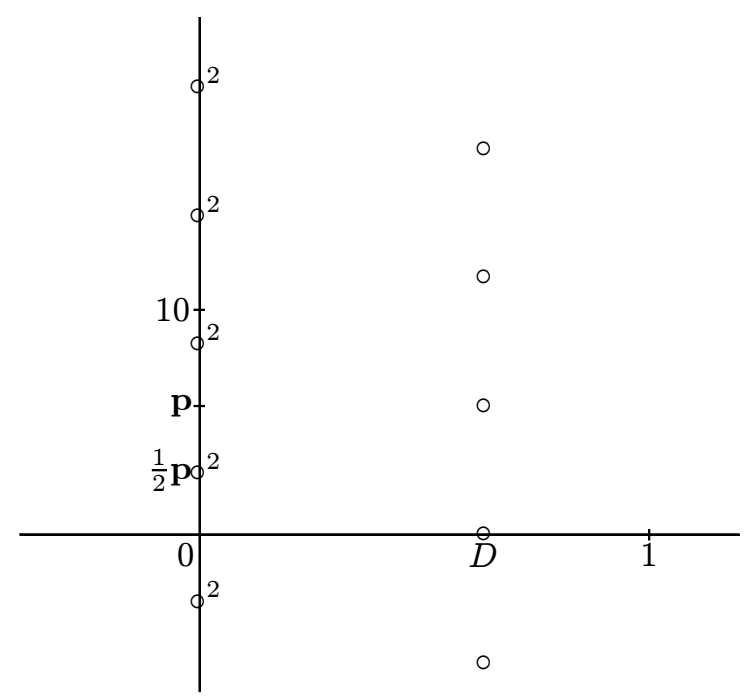

FIGURE 3. The complex roots of a Dirichlet polynomial equation with multiple roots. $D=\log _{3} 2$ and $\mathbf{p}=2 \pi / \log 3$. The symbol $\circ^{2}$ denotes a multiple root of order two. the solution $z=1 / 2$, and another line $\mathrm{e}^{4}$ of double roots $\omega=\frac{1}{2} i \mathbf{p}+i n \mathbf{p}(n \in \mathbb{Z})$ corresponding to the double solution $z=-1$.

2.2.4 The 2-3 and 2-3-4 Nonlattice Equations. The above examples are all lattice equations, as defined in Section 2.1. The reader may get the mistaken impression that in general, it is easy to find the complex roots of a Dirichlet polynomial. However, in the nonlattice case, it is practically impossible to obtain complete information about the complex roots. Nevertheless, in the next sections, we will obtain some partial information about the location and the density of the complex roots of a nonlattice Dirichlet polynomial equation. (See, in particular, Theorem 2.5 below.)

We take two scaling factors $r_{1}=1 / 2, r_{2}=1 / 3$, with multiplicities $m_{1}=m_{2}=1$. The Dirichlet polynomial

$$
1-2^{-s}-3^{-s}
$$

with these scaling factors is nonlattice. Indeed, the weights $w_{1}=\log 2, w_{2}=\log 3$ are not integer multiples of a common generator. The complex roots are found by solving the transcendental equation

$$
2^{-\omega}+3^{-\omega}=1 \quad(\omega \in \mathbb{C}) .
$$

In particular, we find by numerical approximation that $D \approx .78788 \ldots$. See Figure 4 for a diagram of the complex roots.

Let us now consider the case of three scaling factors $r_{1}=1 / 2, r_{2}=1 / 3$ and $r_{3}=1 / 4$, each with multiplicity one. Figure 5 gives the complex roots of the associated nongeneric, nonlattice Dirichlet polynomial

$$
f(s)=1-2^{-s}-3^{-s}-4^{-s} .
$$

The graph below the diagram of the complex roots in Figure 5 gives a plot of the density of the real parts of these roots. It will be explained in more detail in Section 4, Theorem 4.7 and Remark 4.13. We observe the interesting phenomenon that the complex roots of the nongeneric nonlattice equation $f(s)=0$ tend to be denser at the boundaries $\operatorname{Re} s=1.082$ and $\operatorname{Re} s=-1.731$ of the "critical strip," and around $\operatorname{Re} s=0$. Comparing the complex roots of Figure 4 and Figure 5 more closely, one does indeed observe that each complex root of Figure 4 has its counterpart in Figure 5, in the half strip-Re $s>0$, and extra complex dimensions are found to the left of $\operatorname{Re} s=0$ to bring the density to $\log 4 /(2 \pi)$ instead of $\log 3 /(2 \pi)$ (see Theorem 2.5, Equation (2-19)).

\footnotetext{
${ }^{4}$ When we talk about a "line of roots" in this context, we mean a discrete line, as depicted in Figures 1-3. In the following, for convenience, we will continue using this abuse of language.
} 


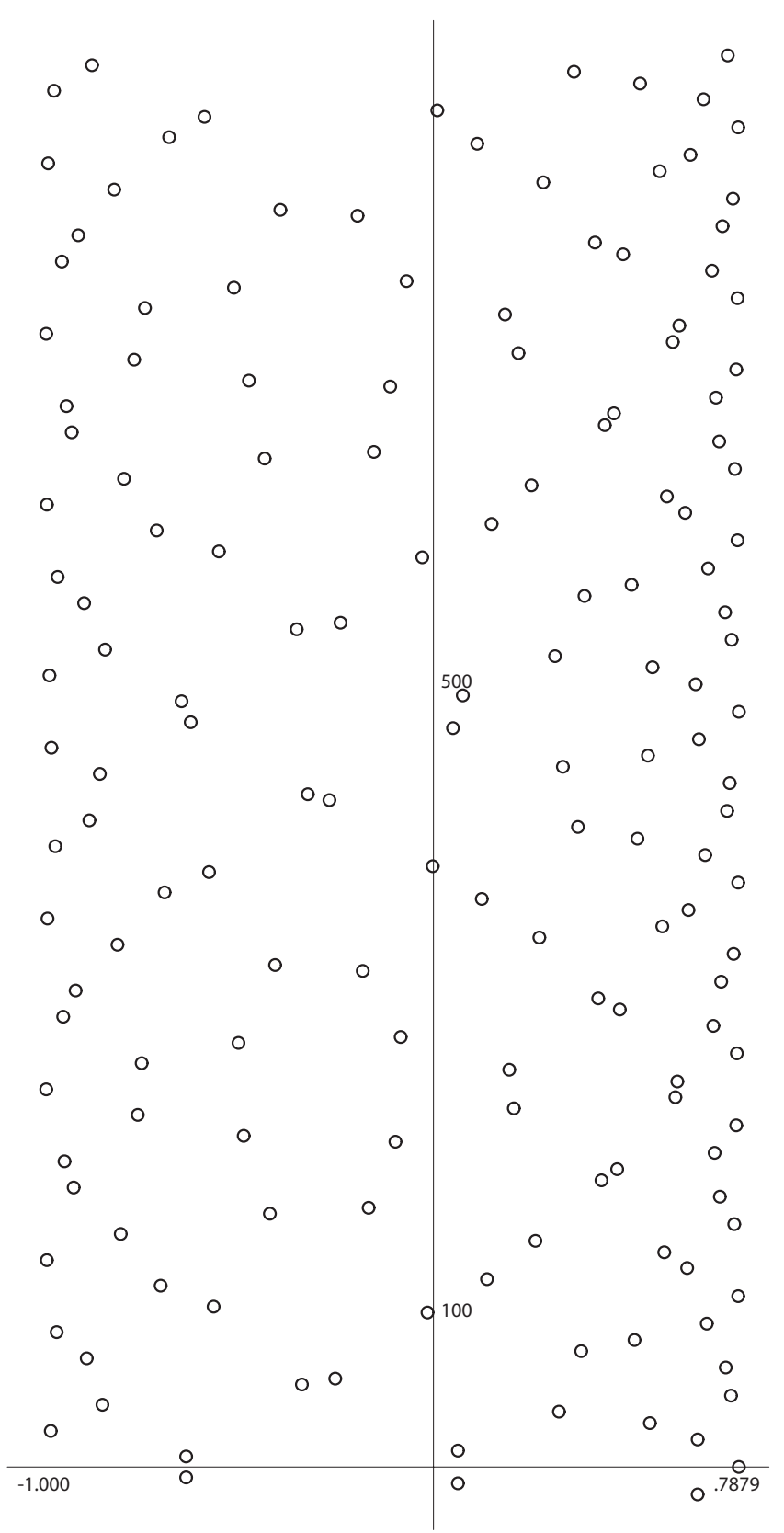

FIGURE 4. The complex roots of the nonlattice Dirichlet polynomial equation $2^{-s}+3^{-s}=1$.

\subsection{The Structure of the Complex Roots}

The simplest example of a Dirichlet polynomial equation is $1-m_{1} r_{1}^{s}=0$; that is, when $M=1$. In this case, the complex roots are $\omega=\left(\log m_{1}\right) / w_{1}+2 k \pi i / w_{1}$, with $k \in \mathbb{Z}$. Hence, the complex roots lie on the vertical line $\operatorname{Re} s=\left(\log \left|m_{1}\right|\right) / w_{1}$, and are separated by $2 \pi i / w_{1}$.

In general, the complex roots of a Dirichlet polynomial equation lie in a strip $D_{l} \leq \operatorname{Re} s \leq D_{r}$, determined as follows: $D_{r}$ is the unique real number such that

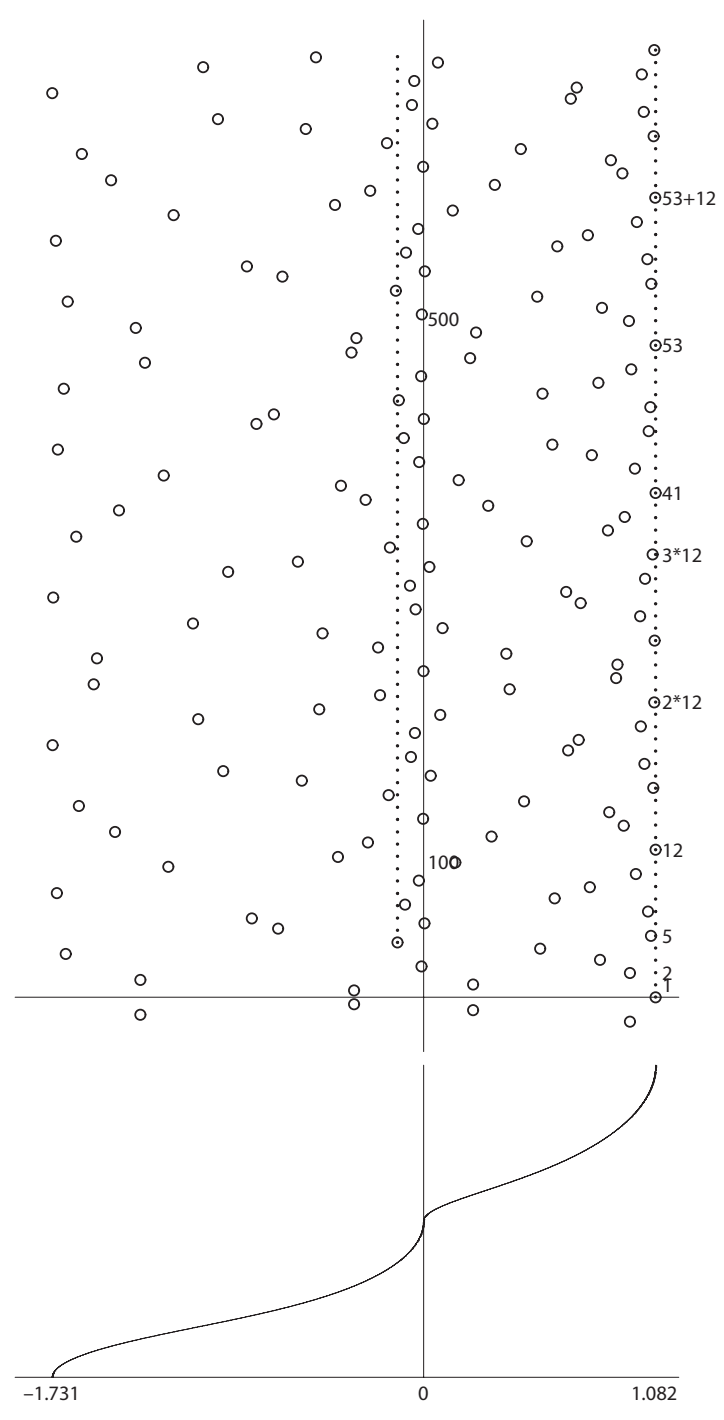

FIGURE 5. The complex roots of the nongeneric nonlattice equation $2^{-s}+3^{-s}+4^{-s}=1$. The accumulative density of the real parts of the complex roots.

$$
\sum_{j=1}^{M}\left|m_{j}\right| r_{j}^{D_{r}}=1
$$

and $D_{l}$ is the unique real number such that

$$
1+\sum_{j=1}^{M-1}\left|m_{j}\right| r_{j}^{D_{l}}=\left|m_{M}\right| r_{M}^{D_{l}} .
$$

The complex roots of the Dirichlet polynomial

$$
f(s)=1-\sum_{j=1}^{M} m_{j} r_{j}^{s}
$$

are described in the following theorem. 
Theorem 2.5. Let $f$ be a Dirichlet polynomial with scaling ratios $1>r_{1}>\cdots>r_{M}>0$ and complex multiplicities $m_{j}$ as above. Then, both in the lattice and the nonlattice case, the set $\mathcal{D}_{f}$ of complex roots of $f$ is contained in the horizontally bounded strip $D_{l} \leq \operatorname{Re} s \leq D_{r}$ :

$$
\mathcal{D}_{f}=\mathcal{D}_{f}(\mathbb{C}) \subseteq\left\{s \in \mathbb{C}: D_{l} \leq \operatorname{Re} s \leq D_{r}\right\} .
$$

It has density $w_{M} /(2 \pi)$ :

$$
\#\left(\mathcal{D}_{f} \cap\{\omega \in \mathbb{C}: 0 \leq \operatorname{Im} \omega \leq T\}\right)=\frac{w_{M}}{2 \pi} T+O(1),
$$

as $T \rightarrow \infty$. Here, the elements of $\mathcal{D}_{f}$ are counted according to multiplicity. If all $m_{j}$ are real, then the set of complex dimensions is symmetric with respect to the real axis. Furthermore, if all multiplicities $m_{j}$ in (2-17) are positive, for $j=1, \ldots, M$, then the value $s=D=D_{r}$ is the only complex root of $f$ on the real line, and it is simple. If, moreover, the multiplicities are integral (i.e., $m_{j} \in \mathbb{N}^{*}$ for $\left.j=1, \ldots, M\right)$, then $D>0$.

In the lattice case, $f(s)$ is a polynomial function of $r^{s}=e^{-w s}$, where $r$ is the multiplicative generator of $f$. So, as a function of $s$, it is periodic with period $2 \pi i / w$. The complex roots $\omega$ are obtained by finding the complex solutions $z$ of the polynomial equation (of degree $k_{M}$ )

$$
\sum_{j=1}^{M} m_{j} z^{k_{j}}=1, \quad \text { with } e^{-w \omega}=z .
$$

Hence there exist finitely many roots $\omega_{1}, \omega_{2}, \ldots, \omega_{q}$, such that

$$
\mathcal{D}_{f}=\left\{\omega_{u}+2 \pi i n / w: n \in \mathbb{Z}, u=1, \ldots, q\right\} .
$$

In other words, the complex roots of $f$ lie periodically on finitely many vertical lines, and on each line they are separated by $2 \pi / w$. The multiplicity of the complex roots corresponding to one value of $z=e^{-w \omega}$ is equal to the multiplicity of $z$ as a solution of (2-20).

In the nonlattice case, the complex roots of $f$ can be approximated (via an explicit procedure specified in Theorem 3.6) by the complex roots of a sequence of lattice equations with larger and larger oscillatory period. Hence, the complex roots of a nonlattice equation have a quasiperiodic structure. Furthermore, there exists a screen $^{5} S$ to the left of the line $\operatorname{Re} s=D$, such that $f$ satisfies $\left(\mathbf{H}_{1}\right)$ and $\left(\mathbf{H}_{2}\right)$ with $\kappa=0$ (see Equations (6-4) and (6-5)), and the complex roots of $f$ in the corresponding window $W$ are simple.

\footnotetext{
${ }^{5}$ See Section 6.
}

Finally, in the generic nonlattice case; i.e., if the weights $w_{j}(j=1, \ldots, M)$ are independent over the rationals, then

$$
D_{l}=\inf \{\operatorname{Re} \omega: \omega \text { is a complex root of } f\}
$$

and

$$
D_{r}=\sup \{\operatorname{Re} \omega: \omega \text { is a complex root of } f\} .
$$

Otherwise, the infimum of the real parts of the complex roots may be larger than $D_{l}$ and the supremum may be smaller than $D_{r}$.

Corollary 2.6. Every integral positive ${ }^{6}$ Dirichlet polynomial has infinitely many complex roots with positive real part.

Proof of Theorem 2.5: For a proof, see [Lapidus and van Frankenhuysen 00, Theorem 2.13, pages 37-40]. There, $D_{l}$ and $D_{r}$ were not introduced, but their property (2-18) can be deduced by an argument similar to that used for $D$. The density estimate $(2-19)$ gives the asymptotic density of the number of roots of $f$, counted with multiplicity. We will prove it here, since the $O(1)$ term improves [Lapidus and van Frankenhuysen 00, Theorem 2.22, page 47].

We need to estimate the winding number of the function $f(s)=1-\sum_{j=1}^{M} m_{j} r_{j}^{s}$ when $s$ runs around a contour $C_{1}+C_{2}+C_{3}+C_{4}$, where $C_{1}$ and $C_{3}$ are vertical segments $c_{1}-i T \rightarrow c_{1}+i T$ and $c_{3}+i T \rightarrow c_{3}-i T$, with $c_{1}>D_{r}$ and $c_{3}<D_{l}$, respectively, and $C_{2}$ and $C_{4}$ are horizontal line segments $c_{1}+i T \rightarrow c_{3}+i T$ and $c_{3}-i T \rightarrow c_{1}-i T$, with $T>0$.

For Re $s=c_{1},\left|\sum_{j=1}^{M} m_{j} r_{j}^{s}\right|<1$, so the winding number along $C_{1}$ is at most $1 / 2$. Likewise, for $\operatorname{Re} s=c_{3}$, we have $\left|1-\sum_{j=1}^{M-1} m_{j} r_{j}^{s}\right| \leq 1+\sum_{j=1}^{M-1}\left|m_{j}\right| r_{j}^{c_{3}}<\left|m_{M}\right| r_{M}^{c_{3}}$, so the winding number along $C_{3}$ is that of $m_{M} r_{M}^{s}$, up to at most $1 / 2$. Hence, the winding number along $C_{1}+C_{3}$ is $(T / \pi) \log r_{M}^{-1}=w_{M} T / \pi$, up to at most 1 .

We will now show that the winding number along $C_{2}+C_{4}$ is bounded, by a classical argument, originally applied to the Riemann zeta function (see [Ingham 92, page 69]). Let $n$ be the number of distinct points on $C_{2}$ at which $\operatorname{Re} f(s)=0$. For real values of $z, 2 \operatorname{Re} f(z+i T)=\sum_{j=1}^{M} m_{j} r_{j}^{z+i T}+\sum_{j=1}^{M} \bar{m}_{j} r_{j}^{z-i T}$. Hence, putting $g(z)=\sum_{j=1}^{M} m_{j} r_{j}^{z+i T}+\sum_{j=1}^{M} \bar{m}_{j} r_{j}^{z-i T}$, we see that $n$ is bounded by the number of zeros of $g$ in a disc containing the interval $\left[D_{l}, D_{r}\right]$. We take the disc centered at $D_{r}+1$, with radius $D_{r}-D_{l}+2$. We have

\footnotetext{
${ }^{6}$ I.e., such that $m_{j} \in \mathbb{N}^{*}$ for $j=1, \ldots, M$.
} 
$\left|g\left(D_{r}+1\right)\right| \geq 2 f\left(D_{r}+1\right)$. Furthermore, let $G$ be the maximum of $g$ on the disc with the same center and radius $e \cdot\left(D_{r}-D_{l}+2\right)$, so $G \leq 2+2 \sum_{j=1}^{M} r_{j}^{D_{r}+1-e\left(D_{r}-D_{l}+2\right)}$. By [Ingham 92, Theorem D, page 49], it follows that $n \leq \log \left|G / g\left(D_{r}+1\right)\right|$. This gives a uniform bound on the winding number over $C_{2}$. The winding number over $C_{4}$ is estimated in the same manner.

The approximation of a nonlattice equation by lattice equations - along with the quasiperiodic structure of the complex roots of a nonlattice equation mentioned at the end of the statement of the theorem-is discussed in Section 3 ; see especially Theorem 3.6 (and the remark following it), which provides more detailed qualitative and quantitative information than in the earlier results obtained in [Lapidus and van Frankenhuysen 00, §2.6].

Remark 2.7. For later reference, we point out the following strip that contains the complex roots. Recall that the weights are ordered in increasing order: $w_{0}=0<w_{1}<$ $\cdots<w_{M}$. Then the horizontally bounded strip

$$
\begin{gathered}
\left\{s \in \mathbb{C}:-\frac{\log \left(1+\sum_{j=1}^{M}\left|m_{j}\right|\right)-\log \left|m_{M}\right|}{w_{M}-w_{M-1}} \leq \operatorname{Re} s\right. \\
\left.\leq \frac{\log \left(1+\sum_{j=1}^{M}\left|m_{j}\right|\right)}{w_{1}}\right\}
\end{gathered}
$$

contains all the complex roots of $f$. This can be seen as follows: A value $s$ cannot be a root of $\sum_{j=0}^{M} m_{j} r_{j}^{s}=0$ if $\left|m_{0} r_{0}^{s}\right|$ is larger than the sum of the other terms. Now $\left|\sum_{j=1}^{M} m_{j} r_{j}^{s}\right| \leq r_{1}^{\sigma} \sum_{j=1}^{M}\left|m_{j}\right|$, provided $\sigma=\operatorname{Re} s \geq 0$. Hence, $\left|\sum_{j=1}^{M} m_{j} r_{j}^{s}\right|<r_{1}^{\sigma} \sum_{j=0}^{M}\left|m_{j}\right|$. The bound on the right is the real solution to the equation $r_{1}^{\sigma} \sum_{j=0}^{M}\left|m_{j}\right|=$ $\left|m_{0}\right| r_{0}^{\sigma}$, taking into account our normalization $w_{0}=0$ and $m_{0}=-1$. Note that this bound is necessarily positive. Similarly, the bound on the left is the real solution to $r_{M-1}^{\sigma} \sum_{j=0}^{M}\left|m_{j}\right|=\left|m_{M}\right| r_{M}^{\sigma}$.

\section{APPROXIMATING A NONLATICE EQUATION BY LATTICE EQUATIONS}

We begin by stating several definitions and a result regarding the convergence of a sequence of analytic functions and of the associated complex roots (i.e., zeroes). Then we study in more detail the particular situation of Dirichlet polynomials.

Definition 3.1. Let $f$ be a holomorphic function on the open set $W \subseteq \mathbb{C}$, and let $\left\{f^{(n)}\right\}_{n=1}^{\infty}$ be a sequence of holomorphic functions on $W^{(n)}$. We say that the $s e$ quence $f^{(n)}$ converges to $f$ (and write $f^{(n)} \rightarrow f$ ) if for every compact set $K \subseteq W$, we have that $K \subseteq W^{(n)}$ for all sufficiently large $n$, and $f^{(n)}(s) \rightarrow f(s)$ uniformly on $K$.

Definition 3.2. Let $f$ be a holomorphic function with set of complex roots $\mathcal{D}=\mathcal{D}(W)$ and let $\left\{f^{(n)}\right\}_{n=1}^{\infty}$ be a sequence of holomorphic functions, with set of complex roots $\mathcal{D}^{(n)}=\mathcal{D}\left(W^{(n)}\right)$. We say that the complex roots of $f^{(n)}$ converge locally to those of $f\left(\right.$ and write $\left.\mathcal{D}^{(n)} \rightarrow \mathcal{D}\right)$, if for every compact set $K \subseteq W$ and every $\varepsilon>0$, there is an integer $n_{0}$ such that for all integers $n \geq n_{0}, K$ is contained in $W^{(n)}$ and there exists a bijection

$$
b_{n}: K \cap \mathcal{D} \rightarrow \mathcal{D}^{(n)}
$$

that respects multiplicities and such that

$$
\left|\omega-b_{n}(\omega)\right|<\varepsilon \text { for all } \omega \in K \cap \mathcal{D} \text {. }
$$

More precisely, $b_{n}$ is a set-valued map from $K \cap \mathcal{D}$ to finite subsets of $\mathcal{D}^{(n)}$, such that the multiplicities of the elements of $b_{n}(\omega)$ add up to the multiplicity of $\omega$ and the distance from $\omega$ to each of the elements of $b_{n}(\omega)$ is bounded by $\varepsilon$.

In the next theorem, we use the notations $f^{(n)} \rightarrow f$ and $\mathcal{D}^{(n)} \rightarrow \mathcal{D}$ of Definitions 3.1 and 3.2. For a proof, see [Lapidus and van Frankenhuysen 00, Theorem 2.26, page 49], with the obvious change of notation.

Theorem 3.3. Let $f$ be a holomorphic function and let $\left\{f^{(n)}\right\}_{n=1}^{\infty}$ be a sequence of holomorphic functions such that $f^{(n)} \rightarrow f$. Then $\mathcal{D}^{(n)} \rightarrow \mathcal{D}$.

We now focus our attention on the case of Dirichlet polynomials. Let scaling ratios $r_{1}, \ldots, r_{M}$ be given that generate a nonlattice Dirichlet polynomial $f$; i.e., by Definition 2.1, the dimension of the $\mathbb{Q}$-vector space generated by the numbers $w_{j}=-\log r_{j}(j=1, \ldots, M)$ is at least 2 .

The following lemma on simultaneous Diophantine approximation can be found in [Schmidt 80], Theorem 1A and the remark following Theorem $1 \mathrm{E}$.

Lemma 3.4. Let $w_{1}, w_{2}, \ldots, w_{M}$ be weights (see Equation (2-1)) such that at least one ratio $w_{j} / w_{1}$ is irrational. Then for every $Q>1$, there exist integers $1 \leq q<Q^{M-1}$ and $k_{1}, \ldots, k_{M}$ such that

$$
\left|q w_{j}-k_{j} w_{1}\right| \leq w_{1} Q^{-1}
$$


for $j=1, \ldots, M$. In particular, $\left|q w_{j}-k_{j} w_{1}\right|<$ $w_{1} q^{-1 /(M-1)}$ for $j=1, \ldots, M$.

Remark 3.5. Note that $\left|q w_{j}-k_{j} w_{1}\right| \neq 0$ when $w_{j} / w_{1}$ is irrational, so that $q \rightarrow \infty$ when $Q \rightarrow \infty$.

Theorem 3.6. Let $f$ be a nonlattice Dirichlet polynomial with weights $w_{0}=0<w_{1}<\cdots<w_{M}$, with multiplicities $m_{0}=-1$ and $m_{1}, \ldots, m_{M} \in \mathbb{C}$, as in $(2-$ 3).Let $Q>1$, and let $q$ and $k_{j}$ be as in Lemma 3.4. Let $\tilde{w}=w_{1} / q$. Then the lattice Dirichlet polynomial $\tilde{f}$ with weights $\tilde{w}_{j}=k_{j} \tilde{w}$ and the same multiplicities $m_{j}$ $(j=1, \ldots, M)$ approximates $f$ in the sense of Definition 3.1. This approximation is such that for every given $\varepsilon>0$, the complex roots of $f$ are approximated up to order $\varepsilon$ for $\varepsilon C Q$ periods of $\tilde{f}$, where

$C=\frac{\sum_{j=1}^{M}\left|m_{j}\right|}{2 \pi}\left(\frac{1+\sum_{j=1}^{M}\left|m_{j}\right|}{\min \left\{1,\left|m_{M}\right|\right\}}\right)^{-2 w_{M} / \min \left\{w_{1}, w_{M}-w_{M-1}\right\}}$

Remark 3.7. Since the number of periods for which this approximation is valid tends to infinity as $Q \rightarrow \infty$, this shows that the complex roots of a nonlattice equation exhibit a quasiperiodic behaviour. This quasiperiodic behaviour evolves in the sense that after following a periodic pattern for a certain (large) number of periods, this pattern starts to disappear, and gradually, a new periodic pattern, belonging to the next value of $q$, begins to emerge. The number of periods for which the pattern belonging to $q$ persists increases with $q$. Since the denominators $q$ increase exponentially as $Q \rightarrow \infty$, every old pattern is only a small fraction (less than one new period) of the new pattern.

Proof of Theorem 3.6: Let $r_{j}=e^{-w_{j}}$ and $\tilde{r}=e^{-\tilde{w}}$. To show that $f$ is well approximated by $\tilde{f}$, we consider the expression

$$
r_{j}^{s}-\tilde{r}^{k_{j} s}=-s \int_{k_{j} \tilde{w}}^{w_{j}} e^{-s x} d x
$$

Using $k_{j} \tilde{w}=\tilde{w}_{j}$ and $\left|w_{j}-\tilde{w}_{j}\right| \leq w_{1} /(q Q)$, we obtain

$$
\begin{aligned}
\left|r_{j}^{s}-\tilde{r}^{k_{j} s}\right| & \leq|s|\left|w_{j}-\tilde{w}_{j}\right| e^{-\sigma w_{j}} \max \left\{1, e^{-\sigma\left(\tilde{w}_{j}-w_{j}\right)}\right\} \\
& \leq|s| \frac{w_{1}}{q Q} e^{|\sigma|\left(w_{M}+w_{1} /(q Q)\right)}
\end{aligned}
$$

for $j=1, \ldots, M$. We simplify this bound further, using $w_{1} /(q Q)<w_{M}$ in the exponent, to find

$$
\begin{aligned}
& |f(s)-\tilde{f}(s)| \\
& \leq \sum_{j=1}^{M}\left|m_{j} r_{j}^{s}-m_{j} \tilde{r}^{k_{j} s}\right| \\
& \leq|s| \frac{w_{1}}{q Q} e^{2 w_{M}|\sigma|} \sum_{j=1}^{M}\left|m_{j}\right| .
\end{aligned}
$$

By $(2-22)$ in Remark 2.7, we may restrict $s=\sigma+i t$ to

$$
\begin{array}{r}
-\frac{\log \left(1+\sum_{j=1}^{M}\left|m_{j}\right|\right)-\log \left|m_{M}\right|}{w_{M}-w_{M-1}} \\
\leq \sigma \leq \frac{\log \left(1+\sum_{j=1}^{M}\left|m_{j}\right|\right)}{w_{1}}
\end{array}
$$

For such $s$,

$$
\begin{aligned}
& |f(s)-\tilde{f}(s)| \\
& \leq|s| \frac{w_{1}}{q Q} \sum_{j=1}^{M}\left|m_{j}\right|\left(\frac{1+\sum_{j=1}^{M}\left|m_{j}\right|}{\min \left\{1,\left|m_{M}\right|\right\}}\right)^{2 w_{M} / \min \left\{w_{1}, w_{M}-w_{M-1}\right\}} \\
& =|s| \frac{w_{1}}{2 \pi q Q} C^{-1} .
\end{aligned}
$$

Thus, if $|s|<\varepsilon C Q \frac{2 \pi q}{w_{1}}$, then $|f(s)-\tilde{f}(s)|<\varepsilon$. Since $\frac{2 \pi i q}{w_{1}}$ is the period of $\tilde{f}$, the theorem follows.

We state the converse, which is of independent interest, but will not be needed in the sequel. For a proof, see [Lapidus and van Frankenhuysen 00, Theorem 2.30, page 51].

Theorem 3.8. Let $f$ be a Dirichlet polynomial, with scaling ratios $r_{0}=1>r_{1}>\cdots>r_{M}>0$ and multiplicities $m_{j} \in \mathbb{C}$. Let $\left\{f^{(n)}\right\}_{n=1}^{\infty}$ be a sequence of Dirichlet polynomials, with scaling ratios $1>r_{1}^{(n)}>\cdots>r_{M^{(n)}}^{(n)}>0$ and multiplicities $m_{j}^{(n)} \in \mathbb{C}$. Let $W=W^{(n)}=\mathbb{C}$ for all $n$. If $f^{(n)} \rightarrow f$, then the scaling ratios converge with the correct multiplicity: for every $\varepsilon>0$, there exists $n_{0}$ such that for all $n \geq n_{0}$ we have: for each $j$, there exists $j^{\prime}$ such that $\left|r_{j}^{(n)}-r_{j^{\prime}}\right|<\varepsilon$, and for each $j^{\prime},\left|\sum_{j} m_{j}^{(n)}-m_{j^{\prime}}\right|<\varepsilon$, where the sum is over those $j$ between 1 and $M^{(n)}$ for which $\left|r_{j}^{(n)}-r_{j^{\prime}}\right|<\varepsilon$.

Note that the statement starts to be interesting for $\varepsilon \leq \min _{1 \leq j<M}\left(r_{j}-r_{j+1}\right) / 2$; that is, when $\varepsilon$ is so small that $\left|r_{j}^{(n)}-r_{j^{\prime}}\right|<\varepsilon$ uniquely determines $j^{\prime}$. Then it says 
that the $r_{j}^{(n)}$ start to cluster around the $r_{j^{\prime}}$ in the sense that for each $j$ there is a unique $j^{\prime}$, and the corresponding multiplicities add up to approximately $m_{j^{\prime}}$.

\subsection{The Computations}

The computations for the nonlattice examples in this paper were done using Maple. The programs can be found on the web page of the second author [van Frankenhuysen 03]. In each case, we approximated the nonlattice equation by a lattice one, resulting in a polynomial equation of degree $d$ between 400 and 5000. Solving the corresponding polynomial equation yields $d$ complex numbers $z$ in an annulus, and the roots $\omega$ are given by $\omega=\log z / \log r+2 k \pi i / \log r$, for $k \in \mathbb{Z}$.

For Figure 4 , we approximated $f(s)=1-2^{-s}-3^{-s}$ by $p(z)=1-z^{306}-z^{485}$, with $z=2^{-s / 306}$ and $r=2^{-1 / 306}$. The corresponding lattice equation with ratios $r^{306}$ and $r^{485}$ has a period of $\mathbf{p}=2 \pi \cdot 306 / \log 2 \approx 2773.8$. For example, the real root $D \approx .7878849110$ of $f$ is approximated by $\tilde{D} \approx D-.1287 \cdot 10^{-5}$. We have $|f(\tilde{D})| \approx .11$. $10^{-5}$ and $|f(\tilde{D}+i \mathbf{p})| \approx .39 \cdot 10^{-2}$. As another example, the root $\tilde{\omega}=.7675115443+45.55415979 i$ approximates a $\operatorname{root} \omega$ of $f$ with an error $\omega-\tilde{\omega} \approx(-.12+.75 i) \cdot 10^{-4}$. Both $\left|p\left(r^{\omega}\right)\right|$ and $|f(\tilde{\omega})|$ are approximately equal to $.64 \cdot 10^{-4}$, and $|f(\tilde{\omega}+i \mathbf{p})|$ approximately equals $.40 \cdot 10^{-2}$. Lemmas 4.2 and 4.10, along with Theorems 4.3, 4.5, and 4.12 in the next sections give theoretical information about the error of approximation.

Since in the applications, we consider only equations with real values for $m_{j}$, the roots come in complex conjugate pairs. Maple normalizes $\log z$ so that the imaginary part lies between $-\pi i$ and $\pi i$. For the density graphs, we took the real parts of the roots $\log z / \log r$ for those $z$ with $-\pi i \leq \operatorname{Im} z \leq 0$, and ordered these values. This way, we obtained a sequence of $(d+1) / 2$ or $d / 2+1$ real parts $v_{1} \leq v_{2} \leq \ldots$ in nondecreasing order. The density graph is a plot of the points $\left(v_{j}, j\right)$ for $1 \leq j \leq d / 2+1$.

Interestingly, if one takes the roots not in one full period, but up to some bound for the imaginary part, the density graph is not smooth, but seems to exhibit a fractal pattern. For $M=2$, this pattern can be predicted from the $\alpha$-adic expansion of $T$; see Section 4.1. This reflects the fact that roots come in quasiperiodic arrays, each one slightly shifted from the previous one, reaching completion only at a period.

The maximal degree 5000 is the limit of computation: It took several hours with our software on a Sun workstation to compute the golden diagram, Figure 8, which involved solving a polynomial equation of degree 4181 . However, finding the roots of the polynomial is the most time-consuming part of the computation. Since these polynomials are "sparse" in the sense that they contain only a few monomials, there may exist ways to speed up this part of the computation.

\section{COMPLEX ROOTS OF A NONLATTICE DIRICHLET POLYNOMIAL}

A nonlattice Dirichlet polynomial has weights $w_{1}<\cdots<$ $w_{M}$, where at least one ratio $w_{j} / w_{1}$ is irrational. Let

$$
f(s)=1-\sum_{j=1}^{M} m_{j} e^{-w_{j} s} .
$$

Assume that all multiplicities $m_{j}$ are positive. Recall from Theorem 2.5 that in this case, $D=D_{r}$ is the unique real solution of the equation $f(s)=0$. Moreover, the derivative

$$
f^{\prime}(s)=\sum_{j=1}^{M} m_{j} w_{j} e^{-w_{j} s}
$$

does not vanish at $D$. We first consider the case $M=2$.

\subsection{Continued Fractions}

We refer the interested reader to [Hardy and Wright 60] for an introduction to the theory of continued fractions, of which we now briefly recall some basic as well as some less well-known elements.

Let $\alpha$ be an irrational real number with a continued fraction expansion

$$
\alpha=\left[\left[a_{0}, a_{1}, a_{2}, \ldots\right]\right]=a_{0}+1 /\left(a_{1}+1 /\left(a_{2}+\ldots\right)\right) .
$$

We recall that the two sequences $a_{0}, a_{1}, \ldots$ and $\alpha_{0}, \alpha_{1}, \ldots$ are defined by $\alpha_{0}=\alpha$ and, for $n \geq 0, a_{n}=$ $\left[\alpha_{n}\right]$, the integer part of $\alpha_{n}$, and $\alpha_{n+1}=1 /\left(\alpha_{n}-a_{n}\right)$. The convergents of $\alpha$,

$$
\frac{p_{n}}{q_{n}}=\left[\left[a_{0}, a_{1}, a_{2}, \ldots, a_{n}\right]\right],
$$

are successively computed by

$$
\begin{aligned}
& p_{-2}=0, p_{-1}=1, p_{n+1}=a_{n+1} p_{n}+p_{n-1}, \\
& q_{-2}=1, q_{-1}=0, q_{n+1}=a_{n+1} q_{n}+q_{n-1} .
\end{aligned}
$$

We also define $q_{n}^{\prime}=\alpha_{1} \cdot \alpha_{2} \cdots \cdots \alpha_{n}$, and note the formula $q_{n+1}^{\prime}=\alpha_{n+1} q_{n}+q_{n-1}$. Then

$$
q_{n} \alpha-p_{n}=\frac{(-1)^{n}}{q_{n+1}^{\prime}} .
$$


For all $n \geq 1$, we have $q_{n} \geq \phi^{n-1}$, where $\phi=(1+\sqrt{5}) / 2$ is the golden ratio.

Let $n \in \mathbb{N}$ and choose $l$ such that $q_{l+1}>n$. We can successively apply division with remainder to compute (see [Ostrowski 22])

$$
n=d_{l} q_{l}+n_{l}, n_{l}=d_{l-1} q_{l-1}+n_{l-1}, \ldots, n_{1}=d_{0} q_{0},
$$

where $d_{\nu}$ is the quotient and $n_{\nu}<q_{\nu}$ is the remainder of the division of $n_{\nu+1}$ by $q_{\nu}$. We set $d_{l+1}=d_{l+2}=\ldots=0$. Then

$$
n=\sum_{\nu=0}^{\infty} d_{\nu} q_{\nu}
$$

We call this the $\alpha$-adic expansion of $n$. Note that $0 \leq d_{\nu} \leq a_{\nu+1}$ and that if $d_{\nu}=a_{\nu+1}$, then $d_{\nu-1}=0$. Also $d_{0}<a_{1}$. It is not difficult to show that these properties uniquely determine the sequence $d_{0}, d_{1}, \ldots$ of $\alpha$-adic digits of $n$.

Lemma 4.1. Let $n$ be given by (4-6). Suppose that the last $k$ digits vanish: $k \geq 0$ is such that $d_{k} \neq 0$ and $d_{k-1}=\cdots=d_{0}=0$. Put $m=\sum_{\nu=k}^{\infty} d_{\nu} p_{\nu}$. Then $n \alpha-m$ lies strictly between

$\frac{(-1)^{k}}{q_{k+1}^{\prime}}\left(d_{k}-1+\alpha_{k+2}^{-1}\right) \quad$ and $\quad \frac{(-1)^{k}}{q_{k+1}^{\prime}}\left(d_{k}+\alpha_{k+2}^{-1}\right)$.

In particular, $n \alpha-m$ lies strictly between $(-1)^{k} / q_{k+2}^{\prime}$ and $(-1)^{k} / q_{k}^{\prime}$.

Proof: We have $n \alpha-m=\sum_{\nu=k}^{\infty} d_{\nu}\left(\alpha q_{\nu}-p_{\nu}\right)$, which is close to the first term $d_{k}(-1)^{k} / q_{k+1}^{\prime}$ by Equation (4-5). Again by this equation, the terms in this sum are alternately positive and negative, and it follows that $n \alpha-m$ lies between the sum of the odd numbered terms and the sum of the even numbered terms. To bound these sums, we use the inequalities $d_{\nu} \leq a_{\nu+1}$ for $\nu>k$. Moreover, $d_{k} \geq 1$, hence $d_{k+1} \leq a_{k+2}-1$. It follows that $n \alpha-m$ lies strictly between

$d_{k}\left(\alpha q_{k}-p_{k}\right)+a_{k+3}\left(\alpha q_{k+2}-p_{k+2}\right)+a_{k+5}\left(\alpha q_{k+4}-p_{k+4}\right)+\ldots$

and

$$
\begin{gathered}
d_{k}\left(\alpha q_{k}-p_{k}\right)+\left(a_{k+2}-1\right)\left(\alpha q_{k+1}-p_{k+1}\right) \\
+a_{k+4}\left(\alpha q_{k+3}-p_{k+3}\right)+\ldots
\end{gathered}
$$

Now $a_{\nu+1}\left(\alpha q_{\nu}-p_{\nu}\right)=\left(\alpha q_{\nu+1}-p_{\nu+1}\right)-\left(\alpha q_{\nu-1}-p_{\nu-1}\right)$, so both sums are telescopic. The first sum evaluates to $d_{k}\left(\alpha q_{k}-p_{k}\right)-\left(\alpha q_{k+1}-p_{k+1}\right)=(-1)^{k}\left(d_{k}+\alpha_{k+2}^{-1}\right) / q_{k+1}^{\prime}$.
The second sum equals $d_{k}\left(\alpha q_{k}-p_{k}\right)-\left(\alpha q_{k+1}-p_{k+1}\right)-$ $\left(\alpha q_{k}-p_{k}\right)=(-1)^{k}\left(d_{k}-1+\alpha_{k+2}^{-1}\right) / q_{k+1}^{\prime}$.

The cruder bounds follow on noting that $1 \leq d_{k} \leq$ $a_{k+1}$, and using $q_{k+2}^{\prime}=\alpha_{k+2} q_{k+1}^{\prime}$ and $a_{k+1}+\alpha_{k+2}^{-1}=$ $\alpha_{k+1}$.

\subsection{Two Generators}

Assume that $M=2$, and let $f$ be defined as in (4-1) with positive multiplicities $m_{1}$ and $m_{2}$ and weights $w_{1}$ and $w_{2}=\alpha w_{1}$, for some irrational number $\alpha>1 .{ }^{7}$ We want to study the complex solutions to the equation $f(\omega)=0$ that lie close to the line $\operatorname{Re} s=D .^{8}$ First of all, such solutions must have $e^{-w_{1} \omega}$ close to $e^{-w_{1} D}$, so $\omega$ will be close to $D+2 \pi i q / w_{1}$, for an integer $q$. We write $\Delta$ for the difference $\omega-D-2 \pi i q / w_{1}$, so that

$$
\omega=D+\frac{2 \pi i q}{w_{1}}+\Delta .
$$

Then we write $\alpha q=p+x(2 \pi i)^{-1}$; hence,

$$
x=2 \pi i(q \alpha-p),
$$

for an integer $p$, which we will specify below. With these substitutions, the equation $f(\omega)=0$ becomes

$$
1-m_{1} e^{-w_{1} D} e^{-w_{1} \Delta}-m_{2} e^{-w_{2} D} e^{-x} e^{-w_{2} \Delta}=0 .
$$

This equation defines $\Delta$ as a function of $x$.

Lemma 4.2. Let $w_{1}, w_{2}>0$ and $\alpha=w_{2} / w_{1}>1$; let $D$ be the real number such that $m_{1} e^{-w_{1} D}+m_{2} e^{-w_{2} D}=1$, and let $\Delta=\Delta(x)$ be the function of $x$, defined implicitly by

$$
m_{1} e^{-w_{1} D} e^{-w_{1} \Delta}+m_{2} e^{-w_{2} D} e^{-x} e^{-w_{2} \Delta}=1,
$$

and $\Delta(0)=0$. Then $\Delta$ is analytic in $x$, in a disc of radius at least $\pi$ around $x=0$, with power series

$$
\begin{gathered}
\Delta(x)=-\frac{m_{2} e^{-w_{2} D}}{f^{\prime}(D)} x+\frac{m_{1} m_{2} w_{1}^{2} e^{-w_{1} D} e^{-w_{2} D}}{2 f^{\prime}(D)^{3}} x^{2} \\
+O\left(x^{3}\right),
\end{gathered}
$$

All the coefficients in this power series are real. Further, the coefficient of $x^{2}$ is positive.

Proof: Write $e^{-w_{1} \Delta}=y(x)$, so that $y$ is defined by $m_{1} e^{-w_{1} D} y+m_{2} e^{-w_{2} D} e^{-x} y^{\alpha}=1$ and $y(0)=1$. Since

\footnotetext{
${ }^{7}$ In the terminology of dynamical systems, this case corresponds to Bernoulli flows; see [Lapidus and van Frankenhuysen 01a, §6.2]

${ }^{8}$ More generally, analogous results can be obtained for the complex solutions close to the vertical line $\operatorname{Re} s=\operatorname{Re} \omega_{0}$, where $\omega_{0}$ is any given complex root of $f$; see, e.g., Remarks 4.4 and 4.11 .
} 
$y$ does not vanish, it follows that if $y(x)$ is analytic in a disc centered at $x=0$, then $\Delta$ will be analytic in that same disc. Moreover, $y$ is real-valued and positive when $x$ is real. Thus, $\Delta$ is real-valued as well when $x$ is real. Further, $y(x)$ is locally analytic in $x$, with derivative

$$
y^{\prime}(x)=\frac{m_{2} e^{-w_{2} D} y^{\alpha} e^{-x}}{m_{1} e^{-w_{1} D}+\alpha m_{2} e^{-w_{2} D} y^{\alpha-1} e^{-x}} .
$$

Hence, there is a singularity at those values of $x$ at which the denominator vanishes, which is at

$$
y=\frac{\alpha}{m_{1} e^{-w_{1} D}(\alpha-1)}
$$

and $e^{-x}=-\alpha^{-\alpha}(\alpha-1)^{\alpha-1} m_{1}^{\alpha} / m_{2}$. Since this latter value is negative, the disc of convergence of the power series for $y(x)$ is

$$
\begin{gathered}
|x|<\mid-\alpha \log \alpha+(\alpha-1) \log (\alpha-1) \\
+\alpha \log m_{1}-\log m_{2}+\pi i \mid .
\end{gathered}
$$

This is a disc of radius at least $\pi$. The first two terms of the power series for $\Delta(x)$ are now readily computed.

Substituting this in $\omega=D+2 \pi i q / w_{1}+\Delta$, we find

$$
\begin{array}{r}
\omega=D+2 \pi i \frac{q}{w_{1}}-\frac{m_{2} e^{-w_{2} D}}{f^{\prime}(D)} x \\
+\frac{m_{1} m_{2} w_{1}^{2} e^{-w_{1} D} e^{-w_{2} D}}{2 f^{\prime}(D)^{3}} x^{2}+O\left(x^{3}\right),
\end{array}
$$

as $x=2 \pi i(q \alpha-p) \rightarrow 0$. We view this formula as expressing $\omega$ as an initial approximation $D+2 \pi i q / w_{1}$, which is corrected by each additional term in the power series. The first corrective term is in the imaginary direction, as are all the odd ones, and the second corrective term, along with all the even ones, is in the real direction. The second term decreases the real part of $\omega$.

Theorem 4.3. Let $\alpha$ be irrational with convergents $p_{\nu} / q_{\nu}$ defined by (4-3) and (4-4). Let $q$ be a positive integer, and let $q=\sum_{\nu=k}^{\infty} d_{\nu} q_{\nu}$ be the $\alpha$-adic expansion of $q$, as in Lemma 4.1. Assume $k \geq 2$, or $k=1$ and $a_{1} \geq 2$, and put $p=\sum_{\nu=k}^{\infty} d_{\nu} p_{\nu}$. Then there exists a complex root of $f$ at

$$
\begin{aligned}
\omega & =D+2 \pi i \frac{q}{w_{1}}-2 \pi i \frac{m_{2} e^{-w_{2} D}}{f^{\prime}(D)}(q \alpha-p) \\
& -2 \pi^{2} \frac{m_{1} m_{2} w_{1}^{2} e^{-w_{1} D} e^{-w_{2} D}}{f^{\prime}(D)^{3}}(q \alpha-p)^{2}+O\left((q \alpha-p)^{3}\right) .
\end{aligned}
$$

The imaginary part of this complex root is approximately $2 \pi i q / w_{1}$, and its distance to the line $\operatorname{Re} s=D$ is at least $C / q_{k+2}^{\prime 2}$, where $C=2 \pi^{2} m_{1} m_{2} w_{1}^{2} e^{-\left(w_{1}+w_{2}\right) D} / f^{\prime}(D)^{3} d e-$ pends only on $w_{1}$ and $w_{2}$ and the multiplicities $m_{1}$ and $m_{2}$.

Moreover, $|f(s)| \gg q_{k+2}^{-2}$ around $s=D+2 \pi i q / w_{1}$ on the line $\operatorname{Re} s=D$, and $|f(s)|$ reaches a minimum of size $C^{\prime}(q \alpha-p)^{2}$, where $C^{\prime}$ depends only on the weights $w_{1}$ and $w_{2}$ and on $m_{1}$ and $m_{2}$.

Proof: By Lemma 4.1, the quantity $q \alpha-p$ lies between $(-1)^{k} / q_{k+2}^{\prime}$ and $(-1)^{k} / q_{k}^{\prime}$. Under the given conditions on $k, q_{k}^{\prime}>q_{k} \geq 2$. Hence $x=2 \pi i(q \alpha-p)$ is less than $\pi$ in absolute value. Then (4-8) gives the value of $\omega$. The estimate for the distance of $\omega$ to the line $\operatorname{Re} s=D$ follows from this formula.

Since the derivative of $f$ is bounded on the line $\operatorname{Re} s=$ $D$, and $f$ does not vanish on this line except at $s=D$, this also implies that $f(s)$ reaches a minimum of order $(q \alpha-p)^{2}$ on an interval around $s=D+2 \pi i q / w_{1}$ on the line $\operatorname{Re} s=D$.

Remark 4.4. An analogous theorem holds for any complex root $\omega_{0}$ (rather than for $D$ ). Indeed, if $f\left(\omega_{0}\right)=0$, then $f\left(\omega_{0}+2 \pi i q / w_{1}\right)$ will be small, and we will find a complex root

$$
\omega=\omega_{0}+2 \pi i \frac{q}{w_{1}}+O(q \alpha-p)
$$

close to $\omega_{0}+2 \pi i q / w_{1}$. This is illustrated in Figures 8 , 11 and 12 below for the repetitions of $D$ and one other complex root.

We obtain more precise information when $q=q_{k}$ and $\omega$ is close to $D+2 \pi i q_{k} / w_{1}$ :

Theorem 4.5. For every $k \geq 0$ (or $k \geq 1$ if $\left.a_{1}=1\right)$, there exists a complex root $\omega$ of $f$ of the form

$$
\begin{aligned}
\omega=D+ & 2 \pi i \frac{q_{k}}{w_{1}}-2 \pi i(-1)^{k} \frac{m_{2} e^{-w_{2} D}}{f^{\prime}(D) q_{k+1}^{\prime}} \\
& -2 \pi^{2} m_{1} m_{2} w_{1}^{2} \frac{e^{-\left(w_{1}+w_{2}\right) D}}{f^{\prime}(D)^{3} q_{k+1}^{\prime 2}}+O\left(q_{k+1}^{\prime-3}\right),
\end{aligned}
$$

as $k \rightarrow \infty$.

Moreover, $|f(s)| \gg q_{k+1}^{\prime-2}$ around $s=D+2 \pi i q_{k} / w_{1}$ on the line $\operatorname{Re} s=D$, and $|f(s)|$ reaches a minimum of size $C^{\prime} q_{k+1}^{\prime-2}$, where $C^{\prime}$ is as in Theorem 4.3. 
Proof: In this case, $q=q_{k}$ is the $\alpha$-adic expansion of $q$. Put $p=p_{k}$. Then $x=2 \pi i(-1)^{k} / q_{k+1}^{\prime}$, which is less than $\pi$ in absolute value. The rest of the proof is the same as the proof of Theorem 4.3 .

Remark 4.6. Theorem 4.3 implies that the density of complex roots in a small strip around $\operatorname{Re} s=D$ is $w_{1} /(2 \pi)$. For "Cantor-like" lattice strings, with $M=1$ (i.e., such that there is only one nonzero weight and $\left.w_{M}=w_{1}\right)$, there is only one line of complex roots, and this density coincides with formula (2-19) of Theorem 2.5. However, it is unclear how wide the strip around $\operatorname{Re} s=D$ should be. For example, in Figure 8, the strip extends to the left of $\operatorname{Re} s=0$.

Theorem 4.7. Let

$$
C=\frac{f^{\prime}(D)^{3 / 2}}{\pi w_{2} \sqrt{2 m_{1} m_{2}}} e^{\left(w_{1}+w_{2}\right) D / 2} .
$$

The density function of the real parts of the complex roots,

$$
\frac{\#\{\operatorname{Re} \omega: \omega \in \mathcal{D}, 0 \leq \operatorname{Im} \omega \leq T, \operatorname{Re} \omega \leq x\}}{\#\{\operatorname{Re} \omega: \omega \in \mathcal{D}, 0 \leq \operatorname{Im} \omega \leq T\}},
$$

has a limit as $T \rightarrow \infty$. The value of this limit is approximated by

$$
1-C \sqrt{D-x}
$$

for values of $x \leq D$ close to $D$.

Proof: By Theorem 2.5, there are approximately $\frac{w_{2}}{2 \pi} T$ complex roots with $0 \leq \operatorname{Im} \omega \leq T$. Given $x<D$, we will count the number of these roots with $\operatorname{Re} \omega>x$. By Theorem 4.3 , for every $q$ with $0 \leq q<\frac{w_{1}}{2 \pi} T$, we find a complex root with $0 \leq \operatorname{Im} \omega \leq T$ and real part approximately $D-C_{1}(q \alpha-p)^{2}$, where

$$
C_{1}=2 \pi^{2} m_{1} m_{2} w_{1}^{2} e^{-\left(w_{1}+w_{2}\right) D} f^{\prime}(D)^{-3} .
$$

By Lemma 4.1, for $q=\sum_{\nu=k}^{\infty} d_{\nu} q_{\nu},|q \alpha-p|$ is roughly equal to $d_{k} / q_{k+1}^{\prime}$, so we need to count the number of $q$ such that (roughly) $d_{k} / q_{k+1}^{\prime}<\sqrt{(D-x) / C_{1}}$. Determine $l$ such that $1 / q_{l+1}^{\prime}<\sqrt{(D-x) / C_{1}} \leq 1 / q_{l}^{\prime}$. Thus, we want those $q$ with $k \geq l$, and if $k=l$, we want $d_{l}<q_{l+1}^{\prime} \sqrt{(D-x) / C_{1}}$.

We find $k \geq l$ with a frequency of $\left(a_{l+1}+1\right)$ times in every $q_{l+1}$ numbers. Indeed, of all $q$ with $0 \leq q<q_{l+1}$, we have $k \geq l$ only for $q=0, q_{l}, 2 q_{l}, \ldots, a_{l+1} q_{l}$. Of these multiples of $q_{l}$, moreover, we want those where the multiple $d_{l}$ is at most $q_{l+1}^{\prime} \sqrt{(D-x) / C_{1}}$. Since $q_{l+1}^{\prime} \approx q_{l+1}$, the

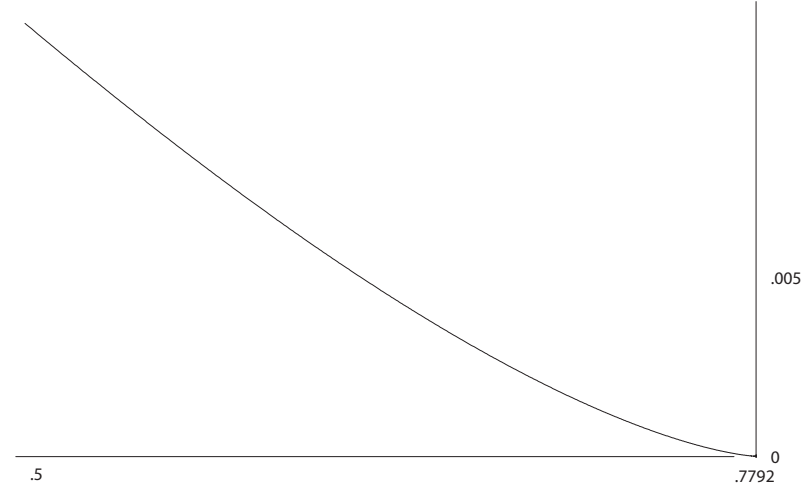

FIGURE 6. The error in the prediction of Theorem 4.7, for the golden Dirichlet polynomial equation $2^{-s}+2^{-\phi s}=1$.

fraction of such numbers $q$ is $\sqrt{(D-x) / C_{1}}$. Hence, in $\frac{w_{1}}{2 \pi} T$ numbers, we find approximately $\frac{w_{1}}{2 \pi} T \sqrt{(D-x) / C_{1}}$ values of $q$ for which $\operatorname{Re} \omega>x$. In a total of $\frac{w_{2}}{2 \pi} T$ complex roots, this is a density of

$$
\left(w_{1} / w_{2}\right) \sqrt{(D-x) / C_{1}},
$$

which is $C \sqrt{D-x}$, where $C$ is given by $(4-11)$. The density of $\omega$ with $\operatorname{Re} \omega \leq x$ is then asymptotically given by $1-C \sqrt{D-x}$.

Remark 4.8. This theorem is illustrated in the diagrams at the bottom of Figures 8 and 11. These diagrams show in one figure the graph of the accumulated density function (4-12) and the graph of the function (4-13). The function (4-13) approximates the accumulative density only in a small neighborhood of $D$. Figure 6 gives a graph of the difference of the two graphs in Figure 8 for the complex roots with real parts between $1 / 2$ and $D$.

\subsection{More than Two Generators}

In this case, the construction of approximations $p_{j} / q$ of $w_{j}$, for $j=1, \ldots, M$, is much less explicit than for $M=2$ since there does not exist a continued fraction algorithm for simultaneous Diophantine approximation. We use Lemma 3.4 as a substitute for this algorithm. The number $Q$ plays the role of $q_{k+1}^{\prime}$ in Theorem 4.5 above. In particular, if $q$ is often much smaller than $Q$, then $w_{1}, \ldots, w_{M}$ is well approximable by rationals, and we find a small root-free region.

Remark 4.9. The $\mathrm{L}^{3}$-algorithm of [Lenstra et al. 82] allows one to find good denominators. However, the problem of finding the best denominator is NP-complete [Lagarias 85, Theorem C]. See also [Rössner and Schnorr] and the references therein, in particular [Hastad et al. 
89]. Also, it may be possible to adapt the algorithm in [Elkies 00] to solve Dirichlet polynomial equations.

Again, we are looking for a solution of $f(\omega)=0$ close to $D+2 \pi i q / w_{1}$, where $f$ is defined by $(4-1)$. We write $\omega=D+2 \pi i q / w_{1}+\Delta$ and

$$
w_{j} q=w_{1} p_{j}+\frac{w_{1}}{2 \pi i} x_{j},
$$

for $j=1, \ldots, M$. For $j=1$, we take $p_{1}=q$ and consequently, $x_{1}=0$. In general,

$$
x_{j}=2 \pi i\left(q w_{j} / w_{1}-p_{j}\right) .
$$

Then $f(\omega)=0$ is equivalent to

$$
\sum_{j=1}^{M} m_{j} e^{-w_{j} D} e^{-x_{j}-w_{j} \Delta}=1 .
$$

The following lemma is the several variables analogue of Lemma 4.2. In the present case, however, we do not know the radius of convergence with respect to the variables $x_{2}, \ldots, x_{M}$.

Lemma 4.10. Let $0<w_{1}<w_{2}<\cdots<w_{M}$, let $D$ be the real number such that $\sum_{j=1}^{M} m_{j} e^{-w_{j} D}=1$, and let $\Delta=\Delta\left(x_{2}, \ldots, x_{M}\right)$ be implicitly defined by

$$
\sum_{j=1}^{M} m_{j} e^{-w_{j} D} e^{-x_{j}-w_{j} \Delta}=1,
$$

with $x_{1}=0$. Then $\Delta$ is analytic in $x_{2}, \ldots, x_{M}$, with power series

$$
\begin{aligned}
& \Delta=-\sum_{j=2}^{M} \frac{m_{j} e^{-w_{j} D}}{f^{\prime}(D)} x_{j}+\frac{1}{2} \sum_{j=2}^{M} \frac{m_{j} e^{-w_{j} D}}{f^{\prime}(D)} x_{j}^{2} \\
& -\frac{1}{2} \sum_{j, k=2}^{M}\left(\frac{f^{\prime \prime}(D)}{f^{\prime}(D)^{3}}+\frac{w_{j}+w_{k}}{f^{\prime}(D)^{2}}\right) m_{j} m_{k} e^{-\left(w_{j}+w_{k}\right) D} x_{j} x_{k} \\
& +O\left(\sum_{j=2}^{M}\left|x_{j}\right|^{3}\right) .
\end{aligned}
$$

This power series has real coefficients. Moreover, the terms of degree two yield a positive definite quadratic form.

Proof: The proof is analogous to that of Lemma 4.2. The positive definiteness of the quadratic form follows from the fact that the complex roots lie to the left of $\operatorname{Re} s=D$; see Theorem 2.5. It can also be verified directly.
We substitute formula (4-15) into $\omega=D+2 \pi i q / w_{1}+$ $\Delta$ to find

$$
\begin{aligned}
& \omega=D+2 \pi i \frac{q}{w_{1}}-\sum_{j=2}^{M} \frac{m_{j} e^{-w_{j} D}}{f^{\prime}(D)} x_{j} \\
& +\frac{1}{2} \sum_{j=2}^{M} \frac{m_{j} e^{-w_{j} D}}{f^{\prime}(D)} x_{j}^{2} \\
& -\frac{1}{2} \sum_{j, k=2}^{M}\left(\frac{f^{\prime \prime}(D)}{f^{\prime}(D)^{3}}+\frac{w_{j}+w_{k}}{f^{\prime}(D)^{2}}\right) m_{j} m_{k} e^{-\left(w_{j}+w_{k}\right) D} x_{j} x_{k} \\
& +O\left(\sum_{j=2}^{M}\left|x_{j}\right|^{3}\right),
\end{aligned}
$$

where $x_{j}=2 \pi i\left(q w_{j} / w_{1}-p_{j}\right)$, for $j=2, \ldots, M$. Again, this formula expresses $\omega$ as an initial approximation $D+$ $2 \pi i q / w_{1}$, which is corrected by each term in the power series. The corrective terms of degree one are again in the imaginary direction, as are all the odd degree ones, and the corrective terms of degree two, along with all the even ones, are in the real direction. The degree two terms decrease the real part of $\omega$.

Remark 4.11. As in Remark 4.4, we have a formula analogous to (4-16) corresponding to any complex root $\omega_{0}$. Thus, every complex root $\omega_{0}$ gives rise to a sequence of complex roots close to the points $\omega_{0}+2 \pi i q / w_{1}$.

Theorem 4.12. Let $M \geq 2$ and let $w_{1}, \ldots, w_{M}$ be weights of a nonlattice equation. Let $Q$ and $q$ be as in Lemma 3.4. Then $f$ has a complex root close to $D+2 \pi i q / w_{1}$ at a distance of at most $O\left(Q^{-2}\right)$ from the line $\operatorname{Re} s=D$, as $Q \rightarrow \infty$. The function $|f|$ reaches a minimum of order $Q^{-2}$ on the line $\operatorname{Re} s=D$ around the point $s=D+$ $2 \pi i q / w_{1}$.

Proof: Again, for $j=2, \ldots, M$, the numbers $x_{j}$ are purely imaginary, so the terms of degree 1 (and of every odd degree) give a correction in the imaginary direction, and only the terms of even degree will give a correction in the real direction. Since $\left|x_{j}\right|<2 \pi / Q$, the theorem follows.

Remark 4.13. By analogy with Theorem 4.7, we find $1-C(D-x)^{(M-1) / 2}$ as an approximation of the density function of the complex roots close to $x=D$, for some positive constant $C$. However, in this case, we do not know the value of $C$. It may depend on the properties of Diophantine approximation of the weights $w_{1}, \ldots, w_{M}$. 


$$
L=1
$$

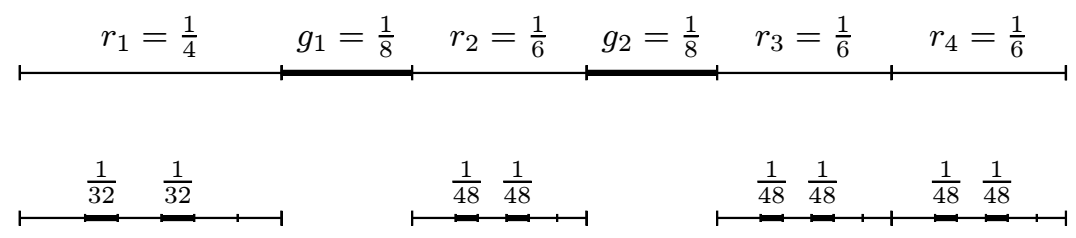

FIGURE 7. An example of a self-similar string (with four scaling ratios $r_{1}=\frac{1}{4}, r_{2}=r_{3}=r_{4}=\frac{1}{6}$, and two gaps $g_{1}=g_{2}=\frac{1}{8}$ ).

Figure 12 suggests that the accumulated density function of the "Two-Three-Five equation" $(M=3$, so $(M-1) / 2=1)$ is approximated by $1-C(D-x)$, for some positive constant $C$.

\section{SELF-SIMILAR FRACTAL STRINGS}

Given an open interval $I$ of length $L$, we construct a selfsimilar fractal string $\mathcal{L}$ with scaling ratios $r_{1}, r_{2}, \ldots, r_{N}$ (see [Frantz 01, Frantz 04, Lapidus 93, Lapidus and van Frankenhuysen 99] and [Lapidus and van Frankenhuysen 00, Chapter 2]). This construction is reminiscent of the construction of the Cantor set. Let $N$ scaling factors $r_{1}, r_{2}, \ldots, r_{N}$ be given $(N \geq 2)$, with

$$
1>r_{1} \geq r_{2} \geq \ldots \geq r_{N}>0 \text {. }
$$

We also define gaps $g_{1}, \ldots, g_{K}\left(K \geq 1, g_{j}>0\right.$ for $j=$ $1, \ldots, K)$ such that

$$
\sum_{j=1}^{N} r_{j}+\sum_{k=1}^{K} g_{k}=1 .
$$

Moreover, we assume that the numbers $r_{j}$ are repeated according to multiplicity. This is different from our earlier convention. Thus, if $\hat{r}_{1}, \ldots, \hat{r}_{M}$ denote the distinct values of the scaling ratios, and $m_{j}$ is the multiplicity of $\hat{r}_{j}$ for $j=1, \ldots, M$, then $N=\sum_{j=1}^{M} m_{j}$. Note that $m_{j}$ is the number of indices $\nu, \nu=1, \ldots, N$, such that $r_{\nu}=\hat{r}_{j}$.

Subdivide $I$ into intervals of length $r_{1} L, \ldots, r_{N} L$, with gaps $g_{1} L, \ldots, g_{K} L$ (see Figure 7 ). The $K$ gaps form the first lengths $g_{1} L, \ldots, g_{K} L$ of the string. Repeat this process with the remaining intervals, to obtain $N K$ new lengths $g_{1} r_{1} L, \ldots, g_{K} r_{N} L$ in the next step, and $N^{k-1} K$ new lengths in the $k$-th step. As a result, we obtain a self-similar fractal string $\mathcal{L}=\left\{l_{j}\right\}$ consisting of intervals of length $l_{j}$ given by

$$
L g_{k} r_{\nu_{1}} r_{\nu_{2}} \ldots r_{\nu_{l}}
$$

for $k=1, \ldots, K$ and all choices of $l \in \mathbb{N}$ and $\nu_{1}, \ldots, \nu_{l} \in$ $\{1, \ldots, N\}$. (We refer the interested reader to the introduction of this paper for a brief discussion of fractal strings, or to [Lapidus and van Frankenhuysen 00, Chapters 1 and 2] for more detailed information.)

Remark 5.1. Throughout this paper, we always assume that a self-similar string is nontrivial; that is, we exclude the trivial case when $\mathcal{L}$ is composed of a single interval. This permits us to avoid having to consider separately this obvious exception to some of our theorems.

Theorem 5.2. Let $\mathcal{L}$ be a self-similar string, constructed as above with scaling ratios $r_{1}, \ldots, r_{N}$. Then the geometric zeta function of this string (see Equation (1-5)) has a meromorphic continuation to the whole complex plane, given by

$$
\zeta_{\mathcal{L}}(s)=\frac{L^{s} \sum_{k=1}^{K} g_{k}^{s}}{1-\sum_{j=1}^{N} r_{j}^{s}}, \quad \text { for } s \in \mathbb{C} .
$$

Here, $L=\zeta_{\mathcal{L}}(1)$ is the total length of $\mathcal{L}$, which is also the length of $I$, the initial interval from which $\mathcal{L}$ is constructed.

Proof: Indeed, for each $k=1, \ldots, K$, we have

$$
\begin{aligned}
\sum_{\nu_{1}=1}^{N} \ldots \sum_{\nu_{l}=1}^{N}\left(g_{k} r_{\nu_{1}} \ldots r_{\nu_{l}}\right)^{s} & =g_{k}^{s} \sum_{\nu_{1}=1}^{N} \ldots \sum_{\nu_{l}=1}^{N} r_{\nu_{1}}^{s} \ldots r_{\nu_{l}}^{s} \\
& =g_{k}^{s}\left(\sum_{j=1}^{N} r_{j}^{s}\right)^{l}
\end{aligned}
$$


Let $s=D$ be the unique real solution of $\sum_{j=1}^{N} r_{j}^{s}=1$. For $\operatorname{Re} s>D$, we have $\left|\sum_{j=1}^{N} r_{j}^{s}\right|<1$. Hence, in view of $(1-5)$ and $(5-3)$, we deduce that

$$
\begin{aligned}
\zeta_{\mathcal{L}}(s) & =\sum_{k=0}^{K} \sum_{l=0}^{\infty}\left(\sum_{\nu_{1}=1}^{N} \ldots \sum_{\nu_{l}=1}^{N}\left(L g_{k} r_{\nu_{1}} \ldots r_{\nu_{l}}\right)^{s}\right) \\
& =L^{s} \sum_{k=1}^{K} g_{k}^{s} \sum_{l=0}^{\infty}\left(\sum_{j=1}^{N} r_{j}^{s}\right)^{l} .
\end{aligned}
$$

Thus, we obtain (5-4) for $\operatorname{Re} s>D$. Since the righthand side of $(5-4)$ is meromorphic on $\mathbb{C}$, we deduce the theorem.

Corollary 5.3. The set of complex dimensions $\mathcal{D}_{\mathcal{L}}$ of the self-similar string $\mathcal{L}$ is the set of solutions of the equation

$$
\sum_{j=1}^{N} r_{j}^{\omega}=1, \quad \omega \in \mathbb{C} .
$$

Moreover, the multiplicity of the complex dimension $\omega$ in $\mathcal{D}_{\mathcal{L}}$ (that is, the multiplicity of $\omega$ as a pole of $\left.\zeta_{\mathcal{L}}\right)$ is equal to the multiplicity of $\omega$ as a solution of (5-5).

Remark 5.4. A self-similar string $\mathcal{L}$ defines a self-similar set in $\mathbb{R}$, namely its boundary $\partial \mathcal{L}$. As is well known (see, e.g., [Falconer 90, Theorems 9.1, 9.3, pages 114, 118]), the Minkowski dimension $D$ of a self-similar set (satisfying the so-called "open set condition") coincides with its "similarity dimension" $s$ [Mandelbrot 83], defined as the unique real solution of Equation (5-5).

Remark 5.5. In [Lapidus and van Frankenhuysen 00, Chapter 2], we have considered the case of self-similar strings with a single gap: $K=1$. An arbitrary selfsimilar string, ${ }^{9}$ however, has multiple gaps, as considered by Mark Frantz in [Frantz 01, Frantz 04]. ${ }^{10}$ To go from the situation where $K=1$ in [Lapidus and van Frankenhuysen 00, Chapter 2] to the present one where $K \geq 1$, it suffices to observe that a general selfsimilar string $\mathcal{L}$ with $K$ gaps, as above, can be naturally decomposed into a finite union of $K$ self-similar strings $\mathcal{L}_{k}(k=1, \ldots, K)$ with a single gap (but with the same scaling ratios $r_{1}, \ldots, r_{N}$ as $\mathcal{L}$ itself); namely, with the above notation, the lengths of each $\mathcal{L}_{k}$ are given by (5-3), for fixed $k \in\{1, \ldots, K\}$. Since, obviously, $\zeta_{\mathcal{L}}(s)=\sum_{k=1}^{K} \zeta_{\mathcal{L}_{k}}(s)$, Theorem 5.2 can also be deduced from its counterpart in our earlier work, [Lapidus

\footnotetext{
${ }^{9}$ Corresponding to an arbitrary self-similar set in $\mathbb{R}$ (other than a single interval).

${ }^{10}$ We wish to thank Mark Frantz for pointing out this more general construction.
}

and van Frankenhuysen 00, Theorem 2.3, page 25], recalled in Equation (1-6) above. Moreover, the main result of [Frantz 01] can also be deduced (by our general methods) from those in [Lapidus and van Frankenhuysen 00, Chapters 2 and 6]. In particular, an arbitrary selfsimilar string is Minkowski measurable if and only if it is a nonlattice string, in which case its Minkowski content $\mathcal{M}(D ; \mathcal{L})$ can be computed explicitly in terms of the total length $L$, the scaling ratios $r_{1}, \ldots, r_{N}$, and the gaps $g_{1}, \ldots, g_{K}$.

Remark 5.6. In [Lapidus and van Frankenhuysen 01a, §2], we found an Euler product for $\zeta_{\mathcal{L}}$, coming from a dynamical system associated with the string, also called a selfsimilar flow. (See Section 6.3 below.) The lengths correspond to the periodic orbits of this dynamical system; see [Lapidus and van Frankenhuysen 01a, Remark 2.15].

Remark 5.7. Note that the zeros of the geometric zeta function $\zeta_{\mathcal{L}}(s)$ given by Equation $(5-4)$ are the solutions of the Dirichlet polynomial equation

$$
\sum_{k=1}^{K} g_{k}^{s}=0, \quad s \in \mathbb{C} .
$$

Hence, the results obtained in our present work can also be applied to understand the structure and approximate the values of the zeros (as well as the poles) of the geometric zeta function of an arbitrary self-similar string. This is noteworthy because when our "explicit formulas" (see Section 6 and [Lapidus and van Frankenhuysen 00, Chapter 4]) are applied in a dynamical context (see [Lapidus and van Frankenhuysen 01a]), the resulting expressions involve both the zeros and poles of $\zeta_{\mathcal{L}}$.

\section{EXPLICIT FORMULAS}

We formulate the explicit formula in the framework of generalized fractal strings, as explained in [Lapidus and van Frankenhuysen 00, Chapter 4]. Let $\eta$ be a measure on $(0, \infty)$, locally bounded and supported away from 0 . The geometric zeta function of $\eta$ is defined by

$$
\zeta_{\eta}(s)=\int_{0}^{\infty} t^{-s} \eta(d t) .
$$

When we take $\eta=\sum_{j=1}^{\infty} \delta_{\left\{l_{j}^{-1}\right\}}$, where $\delta_{\{a\}}$ denotes the Dirac measure concentrated at the point $a$, we find $\zeta_{\eta}(s)=\sum_{j=1}^{\infty} l_{j}^{s}$, and we recover the theory of fractal strings. A special case is when the measure $\eta=$ $\sum_{j=1}^{\infty} \delta_{\left\{l_{j}^{-1}\right\}}$ has a self-similarity property as in [Lapidus 
and van Frankenhuysen 00, §3.4], in which case we recover the theory of self-similar fractal strings.

For a function $\varphi(t)$ on $(0, \infty)$, we let

$$
\widetilde{\varphi}(s)=\int_{0}^{\infty} \varphi(t) t^{s-1} d t
$$

be the Mellin transform of $\varphi$.

We assume that $\zeta_{\eta}$ has a meromorphic continuation to a neighborhood of the set $W$, defined by $W=\{s=$ $\sigma+i t: \sigma \geq S(t)\}$, for some function $S: \mathbb{R} \rightarrow \mathbb{R}$, bounded from above and Lipschitz continuous. ${ }^{11}$ We denote by $\mathcal{D}_{\eta}(W)$ the set of complex dimensions of $\eta$ in $W$, defined as the set of poles of $\zeta_{\eta}$ in $W$ :

$$
\mathcal{D}_{\eta}(W)=\left\{\omega \in W: \zeta_{\eta} \text { has a pole at } \omega\right\} .
$$

We assume the following growth conditions:

There exist real constants $\kappa \geq 0$ and $C>0$ and a sequence $\left\{T_{n}\right\}_{n \in \mathbb{Z}}$ of real numbers tending to $\pm \infty$ as $n \rightarrow \pm \infty$, with $T_{-n}<0<T_{n}$ for $n \geq 1$ and $\lim _{n \rightarrow+\infty} T_{-n} / T_{n}=-1$, such that

$\left(\mathbf{H}_{1}\right)$ For all $n \in \mathbb{Z}$ and all $\sigma \geq S\left(T_{n}\right)$,

$$
\left|\zeta_{\eta}\left(\sigma+i T_{n}\right)\right| \leq C \cdot\left|T_{n}\right|^{\kappa}
$$

$\left(\mathbf{H}_{2}\right)$ For all $t \in \mathbb{R},|t| \geq 1$,

$$
\left|\zeta_{\eta}(S(t)+i t)\right| \leq C \cdot|t|^{\kappa}
$$

With these conditions, we have the following explicit formula for the distribution $\eta$ :

Theorem 6.1. Let $\eta$ be a generalized fractal string, satisfying hypotheses $\left(\mathbf{H}_{1}\right)$ and $\left(\mathbf{H}_{2}\right)$. Then, for suitable functions $\varphi$,

$$
\int_{0}^{\infty} \varphi(t) \eta(d t)=\sum_{\omega \in \mathcal{D}_{\eta}(W)} \operatorname{res}\left(\widetilde{\varphi}(s) \zeta_{\eta}(s) ; \omega\right)+R_{\eta}(\varphi)
$$

where $\omega$ runs through $\mathcal{D}_{\eta}(W)$ and the poles of $\widetilde{\varphi}$, and $R_{\eta}(\varphi)$ is the error term,

$$
R_{\eta}(\varphi)=\frac{1}{2 \pi i} \int_{S} \widetilde{\varphi}(s) \zeta_{\eta}(s) d s .
$$

It is of order $\sup S-1$, in a distributional sense.

See [Lapidus and van Frankenhuysen 00, §4.4] for a proof and discussion of the class of functions $\varphi$ to which

\footnotetext{
${ }^{11}$ In [Lapidus and van Frankenhuysen 99, Lapidus and van Frankenhuysen 01b], the graph of $S$ is referred to as the screen, while the set $W$ is called the window.
}

this formula is applicable. The reader will also find there a discussion of the order of the error term; see, in particular, [Lapidus and van Frankenhuysen 00, Theorems 4.12, 4.20, and 4.23].

We discuss three applications, the geometric counting function, the volume of the tubular neighborhoods, and the counting function of the periodic orbits of a selfsimilar flow. The techniques developed in the present work can be applied to each of these situations to obtain significantly more precise estimates. In order to avoid unnecessary repetitions, we leave it to the interested reader to do so in each particular case.

\subsection{The Geometric Counting Function}

Let

$$
N_{\mathcal{L}}(x)=\#\left\{j: l_{j}^{-1} \leq x\right\}
$$

be the counting function of reciprocal lengths, the geometric counting function. More generally,

$$
N_{\eta}(x)=\int_{0}^{x} \eta(d t)
$$

We take $\varphi(t)=1$ or 0 according to whether $t<x$ and $t>$ $x$, with Mellin transform $\tilde{\varphi}(s)=x^{s} / s$, in formula $(6-6)$ to obtain the explicit formula for the geometric counting function:

$$
N_{\eta}(x)=\sum_{\omega \in \mathcal{D}_{\eta}(W)} \operatorname{res}\left(\frac{x^{s} \zeta_{\eta}(s)}{s} ; \omega\right)+\left\{\zeta_{\eta}(0)\right\}+R_{\eta}(x),
$$

where the term in braces is included if $0 \in W$, but is not a complex dimension of $\eta$. The error term $R_{\eta}(x)$ is of order $x^{\sup S}$.

\subsection{The Volume of the Tubular Neighborhoods}

For

$$
\varphi(t)= \begin{cases}2 \varepsilon & \text { if } t \leq 1 /(2 \varepsilon) \\ 1 / t & \text { if } t>1 /(2 \varepsilon)\end{cases}
$$

with $\widetilde{\varphi}(s)=(2 \varepsilon)^{1-s} /(s(1-s))$, we obtain the volume of the tubular neighborhood

$$
\begin{aligned}
V(\varepsilon)= & \sum_{\omega \in \mathcal{D}_{\eta}(W)} \operatorname{res}\left(\frac{(2 \varepsilon)^{1-s} \zeta_{\eta}(s)}{s(1-s)} ; \omega\right) \\
& +\left\{2 \varepsilon \zeta_{\eta}(0)\right\}+R_{V}(\varepsilon)
\end{aligned}
$$

where the term in braces is included if $0 \in W$, but is not a complex dimension of $\eta$. When the complex dimensions 
are simple, 0 is not a complex dimension, but contained in $W$, and $\eta=\sum_{j=1}^{\infty} \delta_{\left\{l_{j}^{-1}\right\}}$, this reduces to Equation (1-7). The error term in (6-11) is of order $\varepsilon^{1-\sup S}$.

Remark 6.2. There is an interesting analogy between the above formula (or its special case (1-7)) and H. Weyl's formula for the volume of the tubular neighborhoods of a submanifold of $\mathbb{R}^{m}$. The latter formula is a polynomial in $\varepsilon$ whose coefficients are expressed in terms of the Weyl curvature in different (integer) dimensions [Berger and Gostiaux 88, 6.9.8 and Theorem 6.9.9]. In the above explicit formula, $V(\varepsilon)$ is expressed as an expansion in $\varepsilon^{1-\omega}$, where $\omega$ ranges over the "visible" complex dimensions. This suggests that the complex dimensions of fractal strings (and the associated residues ${ }^{12}$ ) could have a direct geometric interpretation. See [Lapidus and van Frankenhuysen 00, Chapter 6] for more information.

\subsection{The Periodic Orbits of Self-Similar Flows}

We refer the interested reader to [Lapidus and van Frankenhuysen 01a], [Parry and Pollicott 83], and [Parry and Pollicott 90], in particular Chapter 6, for more information on the dynamical systems considered here.

Let $N \geq 0$ be an integer and let $\Omega=\{1, \ldots, N\}^{\mathbb{N}}$ be the space of sequences over the alphabet $\{1, \ldots, N\}$. Let $\mathfrak{w}: \Omega \rightarrow(0, \infty]$ be a function, called the weight. On $\Omega$, we have the left shift $\sigma$, given on a sequence $\left(a_{n}\right)$ by $(\sigma a)_{n}=a_{n+1}$. We define the suspended flow $\mathcal{F}_{\mathfrak{w}}$ on the space $[0, \infty) \times \Omega$ as the following dynamical system:

$$
\mathcal{F}_{\mathfrak{w}}(t, a)= \begin{cases}(t, a) & \text { if } 0 \leq t<\mathfrak{w}(a), \\ \mathcal{F}_{\mathfrak{w}}(t-\mathfrak{w}(a), \sigma a) & \text { if } t \geq \mathfrak{w}(a) .\end{cases}
$$

Given a finite sequence $a_{1}, a_{2}, \ldots, a_{l}$, we let $a=$ $a_{1}, a_{2}, \ldots, a_{l}, a_{1}, a_{2}, \ldots, a_{l}, \ldots$ be the corresponding periodic sequence, and

$$
\mathfrak{p}=\left\{a, \sigma a, \sigma^{2} a, \ldots\right\}
$$

the associated finite orbit of $\sigma$, of length $\# \mathfrak{p}$. Note that $\# \mathfrak{p}$ is a divisor of $l$. The total weight of $\mathfrak{p}$ is

$$
\mathfrak{w}_{\text {tot }}(\mathfrak{p})=\sum_{a \in \mathfrak{p}} \mathfrak{w}(a)
$$

We define the dynamical zeta function of $\mathcal{F}_{\mathfrak{w}}$ by the Euler product,

$$
\zeta_{\mathfrak{w}}(s)=\prod_{\mathfrak{p}} \frac{1}{1-e^{-\mathfrak{w}_{\text {tot }}(\mathfrak{p}) s}},
$$

\footnotetext{
${ }^{12}$ Or more generally, the Laurent expansions at these poles if they are not simple.
}

where $\mathfrak{p}$ runs over all finite orbits of $\sigma$. The dynamical complex dimensions of $\mathcal{F}_{\mathfrak{w}}$ are the poles and zeros $\omega$ of $\zeta_{\mathfrak{w}}$, counted without multiplicity. Equivalently, they are the poles of the logarithmic derivative $\zeta_{\mathfrak{w}}^{\prime} / \zeta_{\mathfrak{w}}$. The following function counts the periodic orbits and their multiples by their total weight:

$$
\psi_{\mathfrak{w}}(x)=\sum_{k \mathfrak{w}_{\text {tot }}(\mathfrak{p}) \leq \log x} \mathfrak{w}_{\text {tot }}(\mathfrak{p}) .
$$

It is related to $\zeta_{\mathfrak{w}}$ by

$$
-\frac{\zeta_{\mathfrak{w}}^{\prime}}{\zeta_{\mathfrak{w}}}(s)=\int_{0}^{\infty} x^{-s} d \psi_{\mathfrak{w}}(x),
$$

for $\operatorname{Re} s>D$.

A flow $\mathcal{F}_{\mathfrak{w}}$ is self-similar if $N \geq 2$ and the weight function $\mathfrak{w}$ depends only on the first letter of the sequence on which it is evaluated. We then put

$$
w_{j}=\mathfrak{w}(j, j, j, \ldots),
$$

for $j=1, \ldots, N$. In this case, we have (see [Lapidus and van Frankenhuysen 01a, Theorem 2.10]),

$$
\zeta_{\mathfrak{w}}(s)=\frac{1}{1-\sum_{j=1}^{N} e^{-w_{j} s}} .
$$

Thus, the dynamical complex dimensions coincide with the geometric complex dimensions of the corresponding self-similar string, but they are counted without multiplicity.

Theorem 6.3. (The Prime Orbit Theorem with Error Term.) Let $\mathcal{F}_{\mathfrak{w}}$ be a suspended flow such that $\zeta_{\mathfrak{w}}$ satisfies conditions $\left(\mathbf{H}_{1}\right)$ and $\left(\mathbf{H}_{2}\right)$. Then we have the following equality between distributions:

$$
\begin{gathered}
\psi_{\mathfrak{w}}(x)=\frac{x^{D}}{D}+\sum_{\omega \neq D, 0}-\operatorname{ord}\left(\zeta_{\mathfrak{w}} ; \omega\right) \frac{x^{\omega}}{\omega} \\
+\operatorname{res}\left(-\frac{x^{s} \zeta_{\mathfrak{w}}^{\prime}(s)}{s \zeta_{\mathfrak{w}}(s)} ; 0\right)+R(x),
\end{gathered}
$$

where $\operatorname{ord}\left(\zeta_{\mathfrak{w}} ; \omega\right)$ denotes the order of the zero or pole of $\zeta_{\mathfrak{w}}$ at $\omega$, and

$$
R(x)=-\int_{S} \frac{\zeta_{\mathfrak{w}}^{\prime}}{\zeta_{\mathfrak{w}}}(s) x^{s} \frac{d s}{s}=O\left(x^{\sup S}\right),
$$

as $x \rightarrow \infty$.

If 0 is not a complex dimension of the flow, then the third term on the right-hand side of (6-17) simplifies to $-\zeta_{\mathfrak{w}}^{\prime} / \zeta_{\mathfrak{w}}(0)$. In general, this term is of the form $u+v \log x$, for some constants $u$ and $v$. 
If $D$ is the only complex dimension on the line $\operatorname{Re} s=$ $D$, then the error term,

$$
\sum_{\omega \neq D, 0}-\operatorname{ord}\left(\zeta_{\mathfrak{w}} ; \omega\right) \frac{x^{\omega}}{\omega}+\operatorname{res}\left(-\frac{x^{s} \zeta_{\mathfrak{w}}^{\prime}(s)}{s \zeta_{\mathfrak{w}}(s)} ; 0\right)+R(x),
$$

is estimated by $o\left(x^{D}\right)$, as $x \rightarrow \infty$. If this is the case, then we obtain a Prime Orbit Theorem for $\mathcal{F}_{\mathfrak{w}}$ as follows:

$$
\psi_{\mathfrak{w}}(x)=\frac{x^{D}}{D}+o\left(x^{D}\right)
$$

as $x \rightarrow \infty$.

Remark 6.4. (Geometric and dynamical complex dimensions.) The geometric complex dimensions of a fractal string are defined as the poles of its geometric zeta function. Thus, the complex dimensions are counted with a multiplicity, and the zeros of the geometric zeta function are unimportant. On the other hand, in [Lapidus and van Frankenhuysen 01a], we defined the dynamical complex dimensions as the poles of the logarithmic derivative of the dynamical zeta function. Thus, the dynamical complex dimensions are simple, and both the zeros and the poles of the dynamical zeta function are counted. For self-similar flows, the dynamical zeta function and the geometric zeta function of the corresponding string (without gaps) coincide (up to a normalization), and this zeta function has no zeros. Hence, as sets (without multiplicity), the geometric and dynamical complex dimensions coincide for self-similar flows and strings without gaps.

Remark 6.5. The logarithmic derivative of $\zeta_{\mathfrak{w}}$ for the self-similar flow $\mathcal{F}_{\mathfrak{w}}$ is

$$
\frac{\zeta_{\mathfrak{w}}^{\prime}}{\zeta_{\mathfrak{w}}}(s)=\frac{\sum_{j=1}^{N} w_{j} e^{-w_{j} s}}{1-\sum_{j=1}^{N} e^{-w_{j} s}} .
$$

This corresponds to a self-similar string with "gaps" $g e^{-w_{j}}$, with (in general, noninteger) multiplicity $w_{j}$. The constant $g$ is adjusted so that $(5-2)$ is satisfied: $\sum_{j=1}^{N} e^{-w_{j}}+\sum_{j=1}^{N} g e^{-w_{j}}=1$.

\section{DIMENSION-FREE REGIONS}

We discuss an application of the above results to the theory of self-similar fractal strings, as in Section 5. We use the language of fractal strings, as, for example, in Section 1 and [Lapidus and van Frankenhuysen 00]. That is, a Dirichlet polynomial corresponds to the geometric zeta function of a self-similar string, while the complex roots of such a polynomial correspond to the complex dimensions of this self-similar string.

Definition 7.1. An open domain in the complex plane is a dimension-free region for the string $\mathcal{L}$ if it contains the line $\operatorname{Re} s=D$ and the only pole of $\zeta_{\mathcal{L}}$ in that domain is $s=D$.

We assume that we are in the setting of Section 5 . From Theorem 4.5, and with the notation of Section 4.1 and Section 4.2, we deduce in the case $N=2$,

Corollary 7.2. Assume that the partial quotients $a_{0}, a_{1}, \ldots$ of the continued fraction of $\alpha$ are bounded by a constant C. Put $B=\pi^{4} e^{-\left(w_{1}+w_{2}\right) D} /\left(2 f^{\prime}(D)^{3}\right)$. Then $\mathcal{L}$ has a dimension-free region of the form

$$
\left\{\sigma+i t \in \mathbb{C}: \sigma>D-\frac{B}{C^{2} t^{2}}\right\} .
$$

The function $\zeta_{\mathcal{L}}$ satisfies hypotheses $\left(\mathbf{H}_{1}\right)$ and $\left(\mathbf{H}_{2}\right)$ with $\kappa=2$; see (6-4) and (6-5).

More generally, let $a: \mathbb{R}^{+} \rightarrow[1, \infty)$ be a function such that the partial quotients $\left\{a_{k}\right\}_{k=0}^{\infty}$ of the continued fraction of $\alpha$ satisfy $a_{k+1} \leq a\left(q_{k}\right)$ for every $k \geq 0$. Then $\mathcal{L}$ has a dimension-free region of the form

$$
\left\{\sigma+i t \in \mathbb{C}: \sigma>D-\frac{B}{t^{2} a^{2}\left(t w_{1} /(2 \pi)\right)}\right\} .
$$

If $a(q)$ grows at most polynomially, then $\zeta_{\mathcal{L}}$ satisfies hypotheses $\left(\mathbf{H}_{1}\right)$ and $\left(\mathbf{H}_{2}\right)$ with $\kappa$ such that $t^{\kappa} \geq$ $t^{2} a^{2}\left(t w_{1} /(2 \pi)\right)$, for all $t \in \mathbb{R}$.

Proof: We have $q_{k+1}^{\prime}=\alpha_{k+1} q_{k}^{\prime} \leq 2 a\left(q_{k}\right) q_{k}^{\prime} \leq 4 a\left(q_{k}\right) q_{k}$. By Theorem 4.5, the complex dimension close to $D+$ $i t$ for $t=2 \pi q_{k} / w_{1}$, is located at $D+i\left(t+O\left(q_{k+1}^{\prime-1}\right)\right)-$ $\left(w_{1}^{2} / \pi^{2}\right) B q_{k+1}^{-2}+O\left(q_{k+1}^{\prime-4}\right)$, where the big- $O$ denote realvalued functions. The real part of this complex dimension is less than $D-B t^{-2} a^{-2}\left(t w_{1} /(2 \pi)\right)$.

Example 7.3. One of the simplest nonlattice strings is the golden string, introduced in [Lapidus and van Frankenhuysen 00, page 33]. It is the nonlattice string with $N=2$ and $\alpha=\phi, w_{1}=\log 2$. The continued fraction of $\phi$ is $[[1,1,1, \ldots]]$; hence, $q_{0}, q_{1}, \ldots$ is the sequence of Fibonacci numbers $1,1,2,3,5,8,13, \ldots$, and $q_{k}^{\prime}=\phi^{k}$.

Numerically, we find $D \approx .7792119034$ and the following approximation to the power series $\Delta(x)$ :

$$
\begin{aligned}
& -.47862 x+.08812 x^{2}+.00450 x^{3}-.00205 x^{4} \\
& -.00039 x^{5}+.00004 x^{6}+\ldots
\end{aligned}
$$


For every $k \geq 0$, we find a complex dimension close to $D+2 \pi i q_{k} / \log 2$. For example, $q_{9}=55$, and we find a complex dimension at $D-.00023+498.58 i$. More generally, for numbers that can be written as a sum of Fibonacci numbers of relatively high index, like $q=55+5$ or $q=55-5=34+13+3$, we find a complex dimension close to $D+2 \pi i q / \log 2$. For $q=60$ and $q=50$, we find a complex dimension, respectively, at $D-.023561+543.63 i$ and at $D-.033919+453.53 i$. In both these cases, the distance to the line $\operatorname{Re} s=D$ is comparable to the distance of the complex dimension close to $D+2 \pi i \cdot(5 / \log 2)$ to this line, which is located at $D-.028499+45.05 i$. See Figure 8 , where the markers indicate the Fibonacci numbers, $1,1,2,3,5,8,13,21,34, \ldots$. The pattern persists for other complex dimensions as well. Indeed, every complex dimension repeats itself according to the pattern of the Fibonacci numbers. This is illustrated for one other complex dimension.

The second graph, at the bottom of Figure 8, shows the accumulative density of the real parts of the complex dimensions, compared with the predicted density (graphed for $D_{l} \leq x \leq D$ ) of Theorem 4.7.

Figure 8 was obtained by approximating $\phi$ by $4181 / 2584$. Thus, the figure shows the complex dimensions of the lattice string with generator $w=$ $(\log 2) / 2584, k_{1}=2584, k_{2}=4181$. The oscillatory period of this string is $2 \pi / w \approx 23423.23721$. Figure 9 gives intermediate stages of this approximation.

Example 7.4. In Figure 10, we give a diagram of the complex dimensions of the "golden+" string, defined as the nongeneric nonlattice string with $N=3$ and ratios $2^{-1}, 2^{-\phi}$, and $2^{-2}$. For the real parts, we observe the same phenomenon of "phase transition" in the complex dimensions as discussed in Section 2.2.4.

To produce this diagram, we approximated $1-2^{-s}-$ $2^{-\phi s}-2^{-2 s}$ by $1-z^{2584}-z^{4181}-z^{5168}$, for $z=r^{s}$, $r=2^{-1 / 2584}$.

Example 7.5. Figure 11 gives the complex dimensions and the density of their real parts of the generic nonlattice string with weights $w_{1}=\log 2, w_{2}=\alpha \log 2$, where $\alpha$ is the positive real number with continued fraction $[[1,2,3,4, \ldots]]$. One can compute that

$$
\alpha=\frac{\sum_{n=0}^{\infty} \frac{1}{(n !)^{2}}}{\sum_{n=0}^{\infty} \frac{1}{n !(n+1) !}}=\frac{I_{0}(2)}{I_{1}(2)},
$$

where $I_{k}(z)=\sum_{n=0}^{\infty} \frac{(z / 2)^{2 n+k}}{n !(n+k) !}$ is the modified Bessel function. Indeed, by [Whittaker and Watson 96, pages 359,373$]$, we have the recursion relation,

$$
\frac{I_{n-1}(z)}{I_{n}(z)}=\frac{2 n}{z}+\frac{1}{\frac{I_{n}(z)}{I_{n+1}(z)}},
$$

for $n=1,2, \ldots$.

Thus, $I_{0}(z) / I_{1}(z)=[[2 / z, 4 / z, 6 / z, \ldots]]$ and so $\alpha$ is given by Equation (7-3), as claimed. (See also [Watson 95].) For this reason, we propose to call this string the Bessel string.

Even though $\alpha$ is better approximable by rationals than numbers with bounded partial quotients, qualitatively there does not seem to be a significant difference with the golden string.

Figure 11 is obtained by approximating $\alpha$ by 1393/972. The markers illustrate again the different periodic patterns. The repetitions occur at denominators of convergents of $\alpha$, which are the numbers $1,2,7,30,157, \ldots$, and combinations of these (in the sense of the $\alpha$-adic expansion of Equation (4-6)).

Example 7.6. The Two-Three-Five string is an example with $N>2$. It has $N=3$ scaling ratios $r_{1}=1 / 2, r_{2}=$ $1 / 3, r_{3}=1 / 5$. See Figure 12 for a diagram of the complex dimensions and of the density of their real parts.

Figure 12 is obtained using the approximation $\log _{2} 3 \approx$ $2826 / 1783, \log _{2} 5 \approx 4140 / 1783$.

In the following corollary, given $N \geq 2$, we use the expression $w_{1}, \ldots, w_{N}$ is " $a$-approximable" if $a:[1, \infty) \rightarrow$ $\mathbb{R}^{+}$is an increasing function such that for every $q \geq 1$, $j \in\{1, \ldots, N\}$ and integers $p_{j}$,

$$
\left|q w_{j}-p_{j} w_{1}\right| \geq \frac{w_{1}}{a(q)} q^{-1 /(N-1)} .
$$

From Theorem 4.12, we deduce

Corollary 7.7. Let $N \geq 2$. The best dimension-free region that $\mathcal{L}$ can have is of size

$$
\left\{\sigma+i t \in \mathbb{C}: \sigma \geq D-O\left(t^{-2 /(N-1)}\right)\right\} .
$$

The implied constant depends only on $w_{1}, \ldots, w_{N}$.

If $w_{1}, \ldots, w_{N}$ is "a-approximable," then the dimension-free region has the form

$$
\left\{\sigma+i t \in \mathbb{C}: \sigma \geq D-O\left(a^{-2}\left(w_{1} t /(2 \pi)\right) t^{-2 /(N-1)}\right)\right\} .
$$

Remark 7.8. If $a(q)$ grows faster than polynomially, we obtain a bound of the form $x^{D} / a^{\text {inv }}(\log x)$ for the error in the explicit formula for $V(\varepsilon)$; see $(6-11)$. Here, $a^{\text {inv }}$ is the inverse function of $a$. 


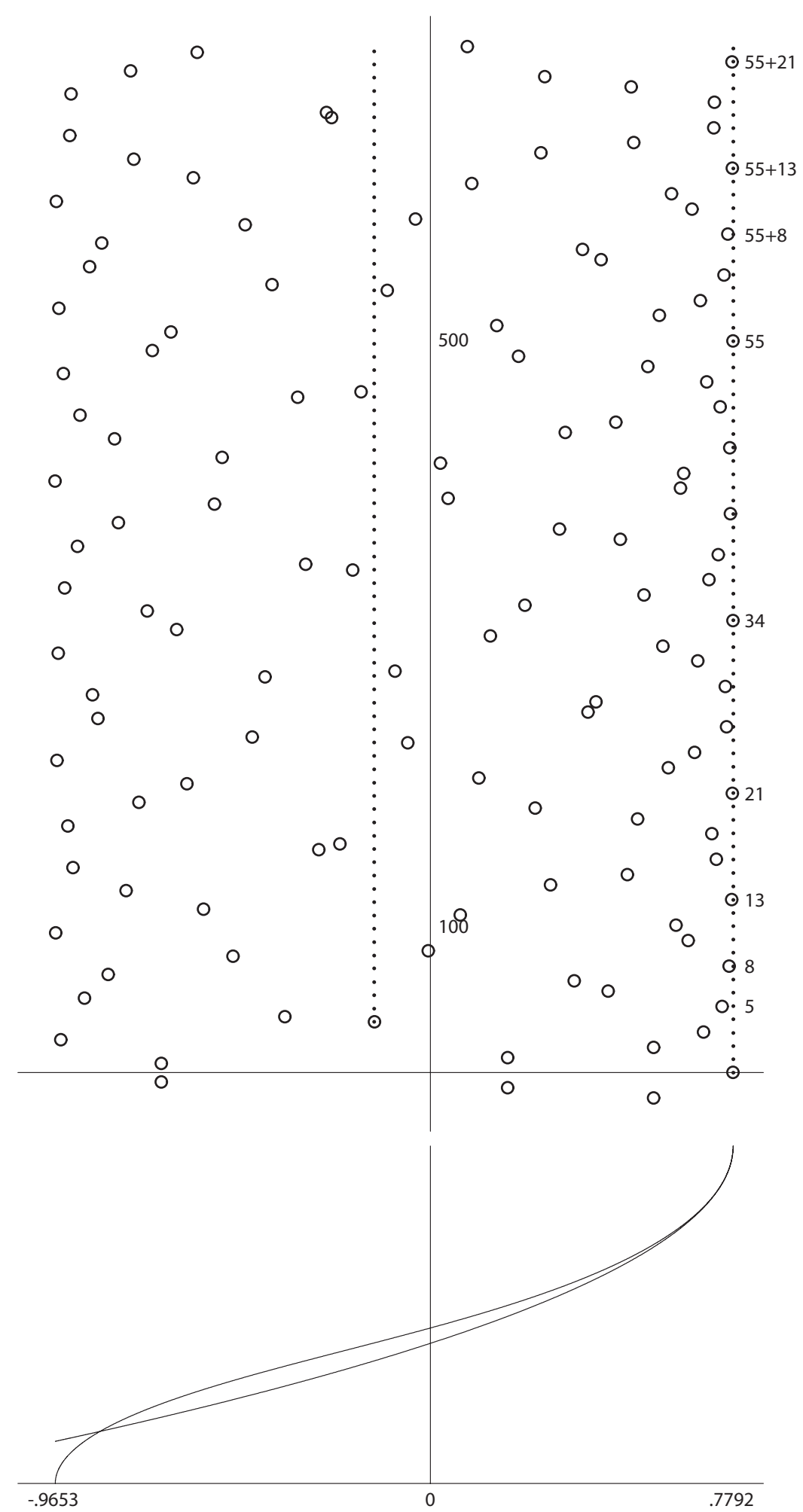

FIGURE 8. The complex dimensions of the golden string; the accumulative density of their real parts, compared with the theoretical prediction of Theorem 4.7 . 

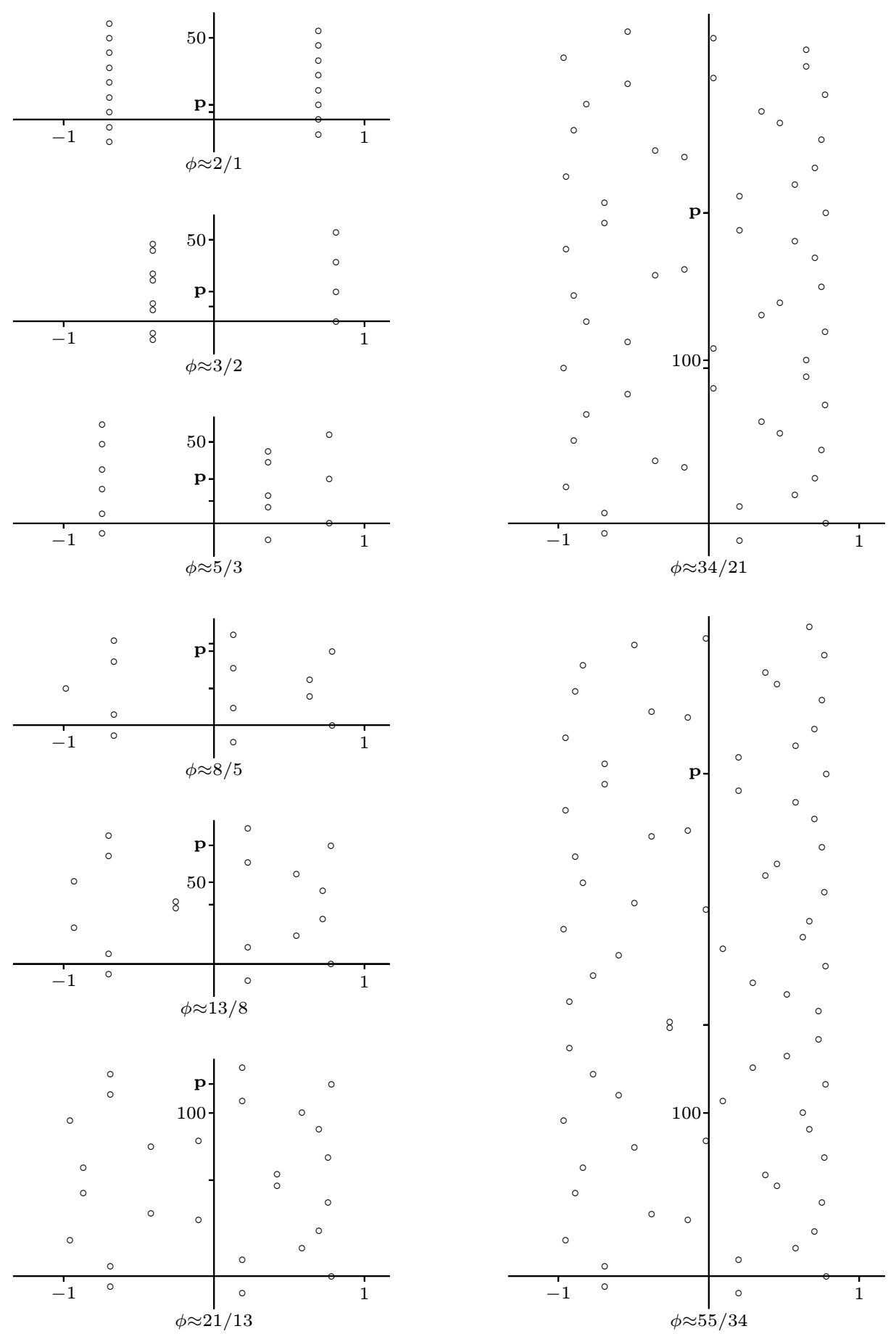

FIGURE 9. Approximations to the complex dimensions of the golden string. Emergence of the quasiperiodic pattern. 


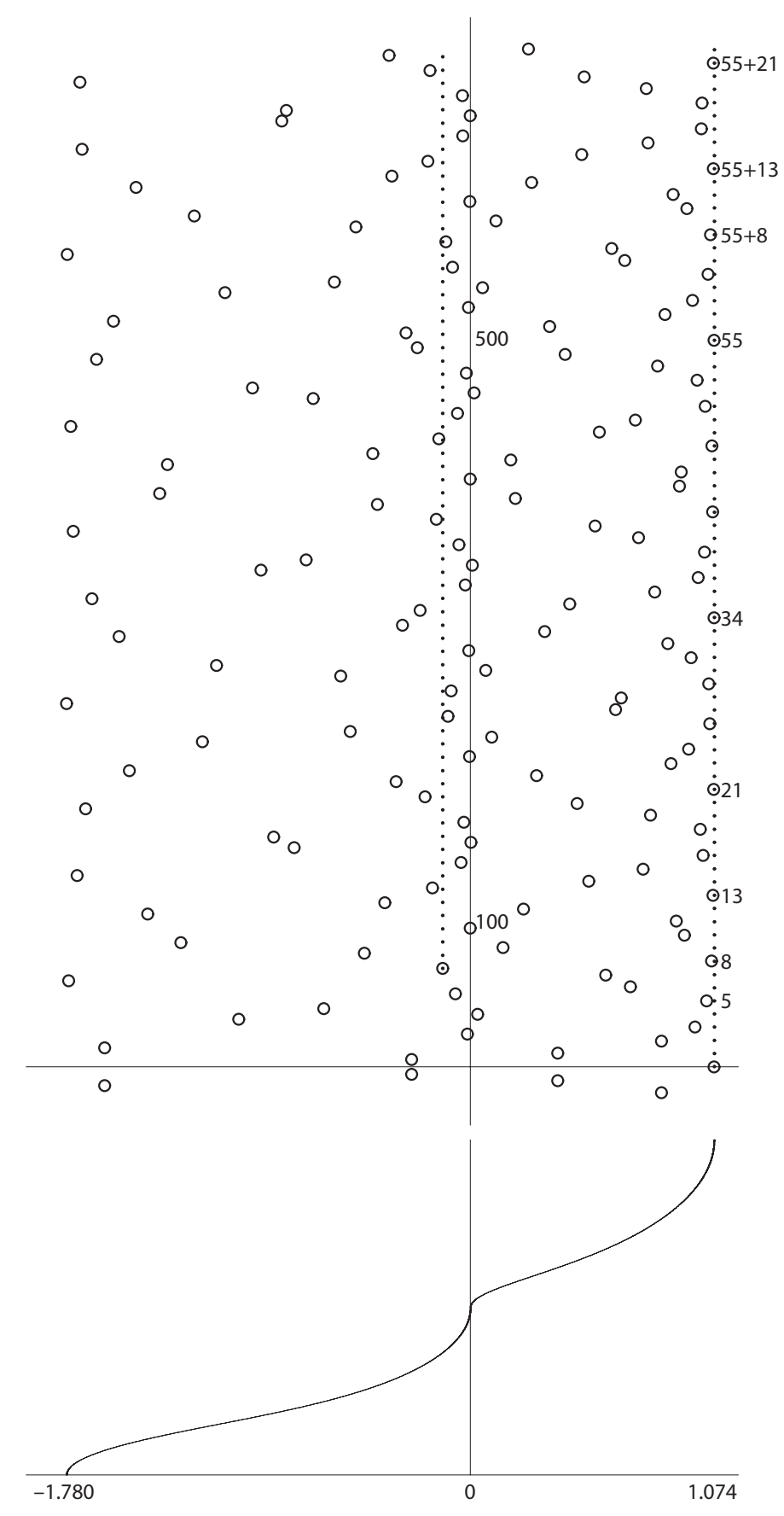

FIGURE 10. The complex dimensions of the golden+ string; the accumulative density of their real parts. 




FIGURE 11. The complex dimensions of the Bessel string; the accumulative density of their real parts, compared with the theoretical prediction of Theorem 4.7. 




FIGURE 12. The quasiperiodic behavior of the complex dimensions of the Two-Three-Five string; the accumulative density of the real parts of the complex dimensions. 
Remark 7.9. The best dimension-free region is obtained when $w_{1}, \ldots, w_{N}$ is badly approximable by rationals; i.e., when the weights are only $a$-approximable for a constant function $a=a(q)$. This corresponds to the case of two generators when $\alpha$ has bounded partial quotients in its continued fraction (Corollary 7.2). When $w_{1}, \ldots, w_{N}$ is better approximable by rationals, the dimension-free region is smaller and there are complex dimensions closer to the line $\operatorname{Re} s=D$. Such nonlattice strings behave more like lattice strings (compare Theorem 2.5).

Remark 7.10. Regarding the dependence on $N$, we see from comparing Corollaries 7.2 and 7.7 that in general, depending of course on the properties of simultaneous Diophantine approximation of the weights, nonlattice strings with a larger number of scaling ratios have a wider (better) dimension-free region.

Remark 7.11. By formulas (1-9) and (1-10) of the introduction, we deduce that for a nonlattice self-similar string, $V(\varepsilon)$ is approximated by its leading term up to an error of order $|\log \varepsilon|^{-(N-1) / 2}$, or worse if the weights are well approximable.

\section{THE REAL PARTS OF THE COMPLEX DIMENSIONS OF A NONLATTICE STRING}

In this section, we obtain a rigorous result and make several conjectures regarding the real parts of the complex dimensions of nonlattice strings. It is noteworthy that both the theorem and the conjectures were themselves suggested by computer experimentation guided by our theoretical investigations.

Recall from Remark 4.11 that the analogue of formula (4-16) holds for any complex dimension $\omega$, besides $D$.

Theorem 8.1. The set of real parts of the complex dimensions of a nonlattice string has no isolated points.

Proof: By formula (4-16), applied for $\omega$, every complex dimension gives rise to a sequence of complex dimensions close to the points $\omega+2 \pi i q / w_{1}$, for integers $q$. Since the corrective terms are not all purely imaginary, we find complex dimensions with real parts close to $\operatorname{Re} \omega$. When $q$ increases through a sequence of integers such that for each $j=1, \ldots, N, x_{j}=2 \pi i\left(q w_{j} / w_{1}-p_{j}\right) \rightarrow 0$, we find a sequence of complex dimensions whose real parts approach $\operatorname{Re} \omega$.
Remark 8.2. Recall from [Lapidus and van Frankenhuysen $00, \S 10.2$ and $\S 10.3]$ that a string is said to be fractal in dimension $\alpha$ if it has a complex dimension with real part $\alpha$. Each complex dimension gives rise to oscillations in the geometry of the fractal string. The frequencies of these oscillations are determined by the imaginary part of the complex dimension, and the real part of the complex dimension determines the amplitude of the oscillations. We define the set of dimensions of fractality of a fractal string as the closure of the set of real parts of its complex dimensions. Thus, Theorem 8.1 can be interpreted as saying that a nonlattice string is fractal in a perfect set of fractal dimensions.

\subsection{The Density of the Real Parts}

The density of the real parts of the complex dimensions of six different nonlattice strings are plotted in Figures 5, 8, 10-13, respectively. These figures show the graph of the function given by formula (4-12) for some large value of $T$. Since there are no horizontal pieces in these graphs, we conjecture that the real parts are dense in $\left[D_{l}, D\right]$. More generally, we expect the same to be true for the real parts of the complex dimensions of any generic nonlattice string.

We summarize this discussion by stating the following conjecture:

Conjecture 8.3. If $\mathcal{L}$ is a generic nonlattice string, the set of dimensions of fractality of $\mathcal{L}$ is equal to the entire interval $\left[D_{l}, D\right]$, where $D_{l}$ is defined in (2-16) and $D$ is the Minkowski dimension of $\mathcal{L}$.

The graphs of densities are qualitatively different for $M=2$ and $M=3$ (i.e., for either two or three different scaling factors): The complex dimensions of the TwoThree-Five string show a "phase transition" between negative and positive real part. (See Figures 10 and 12, where $M=3$, and compare with Figures 8 and 11 where $M=2$.) In the case of the Two-Three-Five string of Example 7.6 (see Figure 12), the density for negative real parts is approximately .77 (i.e., below average density), while for positive real parts, it is 1.08 , slightly above average density. The density around vanishing real part becomes as large as 2.1. It seems that for larger $M$, this phenomenon persists. Therefore, we make the following conjecture:

Conjecture 8.4. As $M \rightarrow \infty$, there exists a vertical line such that the density of the complex dimensions off this line vanishes in the limit. 




FIGURE 13. The complex dimensions of the nonlattice string with $N=4$ and $r_{1}=1 / 2, r_{2}=1 / 3, r_{3}=1 / 5, r_{4}=1 / 7$; the accumulative density of their real parts. 
We close this section with an example of the complex dimensions and the density of the real parts of the generic nonlattice string with $r_{1}=1 / 2, r_{2}=1 / 3, r_{3}=1 / 5$, and $r_{4}=1 / 7$. See Figure 13. As was suggested in Remark 7.10, the complex dimensions tend to be more concentrated in the middle, away from $\operatorname{Re} s=D$ and $\operatorname{Re} s=$ $D_{l}$. We have used the approximation $\log _{2} 3 \approx 699 / 441$, $\log _{2} 5 \approx 1024 / 441$, and $\log _{2} 7 \approx 1238 / 441$.

Clearly, more mathematical experimentation - guided by our theoretical investigations - is needed to determine the generality of this phenomenon and to formulate suitable additional conjectures regarding the fine structure of the complex dimensions of nonlattice strings.

\section{ACKNOWLEDGMENTS}

We thank Traugott Schulmeiß of Jena for providing us with the information on Diophantine approximation given in Remark 4.9. We also wish to thank the two referees of this paper, along with Robert S. Strichartz, for their careful reading of the manuscript and their detailed and helpful comments, which led in particular to a significant improvement in the presentation of our results. This work was partially supported by the National Science Foundation under grant DMS-0070497 (for M.L.L.).

\section{REFERENCES}

[Berger and Gostiaux 88] M. Berger and B. Gostiaux. Differential Geometry: Manifolds, Curves and Surfaces, English translation. Berlin: Springer-Verlag, 1988.

[Elkies 00] N. D. Elkies. "Rational Points Near Curves and Small Nonzero $\left|x^{3}-y^{2}\right|$ via Lattice Reduction." In Proceedings of ANTS-4, edited by W. Bosma, pp. 3363, Lecture Notes in Computer Science 1838, Berlin: Springer-Verlag, 2000.

[Falconer 90] K. J. Falconer. Fractal Geometry: Mathematical Foundations and Applications. Chichester, UK: Wiley, 1990.

[Feller 66] W. Feller. An Introduction to Probability Theory and its Applications, Vol. II. New York: Wiley, 1966.

[Frantz 01] M. Frantz. "Minkowski Measurability and Lacunarity of Self-Similar Sets in $\mathbb{R}$," Preprint, December 2001.

[Frantz 04] M. Frantz. "Lacunarity, Minkowski Content, and Self-Similar Sets in $\mathbb{R}$," To appear in Fractal Geometry and Applications: A Jubilee of Benoit Mandelbrot, edited by M. L. Lapidus and M. van Frankenhuysen, Proc. Symposia in Pure Mathematics, Providence, RI: Amer. Math. Soc., March 2004.
[Hardy and Wright 60] G. H. Hardy and E. M. Wright. An Introduction to the Theory of Numbers. Oxford, UK: Oxford University Press, 1960.

[Hastad et al. 89] J. Hastad, B. Just, J. C. Lagarias, and C. P. Schnorr. "Polynomial Time Algorithms for Finding Integer Relations among Real Numbers." SIAM J. Comput. 18 (1989), 859-881.

[Ingham 92] A. E. Ingham. The Distribution of Prime Numbers. Cambridge, UK: Cambridge University Press, 1992.

[Kigami and Lapidus 93] J. Kigami and M. L. Lapidus. "Weyl's Problem for the Spectral Distribution of Laplacians on p.c.f. Self-Similar Fractals." Commun. Math. Phys. 158 (1993), 93-125.

[Lagarias 85] J. C. Lagarias. "The Computational Complexity of Simultaneous Diophantine Approximation Problems." SIAM J. Comput. 14 (1985), 196-209.

[Lalley 89] S. P. Lalley. "Renewal Theorems in Symbolic Dynamics, with Applications to Geodesic Flows, Noneuclidean Tessellations and their Fractal Limits." Acta Math. 163 (1989), 1-55.

[Lalley 91] S. P. Lalley. "Probabilistic Counting Methods in Certain Counting Problems of Ergodic Theory." In Ergodic Theory, Symbolic Dynamics and Hyperbolic Spaces, edited by T. Bedford, M. Keane, and C. Series, pp. 223258, Oxford, UK: Oxford Univ. Press, 1991.

[Lapidus 93] M. L. Lapidus. "Vibrations of Fractal Drums, the Riemann Hypothesis, Waves in Fractal Media, and the Weyl-Berry Conjecture." In Ordinary and Partial Differential Equations, edited by B. D. Sleeman and R. J. Jarvis, Vol. IV, Proc. Twelfth Intern. Conf. (Dundee, Scotland, UK, June 1992), Pitman Research Notes in Math. Series 289, pp. 126-209, London: Longman Scientific and Technical, 1993.

[Lapidus and Maier 95] M. L. Lapidus and H. Maier. "The Riemann Hypothesis and Inverse Spectral Problems for Fractal Strings." J. London Math. Soc. (2) 52 (1995), $15-34$.

[Lapidus and Pomerance 93] M. L. Lapidus and C. Pomerance. "The Riemann Zeta-Function and the OneDimensional Weyl-Berry Conjecture for Fractal Drums." Proc. London Math. Soc. (3) 66 (1993), 41-69.

[Lapidus and van Frankenhuysen 99] M. L. Lapidus and M. van Frankenhuysen. "Complex Dimensions of Fractal Strings and Oscillatory Phenomena in Fractal Geometry and Arithmetic." Contemporary Mathematics 237 (1999), 87-105.

[Lapidus and van Frankenhuysen 00] M. L. Lapidus and M. van Frankenhuysen. Fractal Geometry and Number Theory (Complex dimensions of fractal strings and zeros of zeta functions), Research Monographs. Boston, MA: Birkhäuser, 2000. (Second revised and enlarged edition to appear in 2004.) 
[Lapidus and van Frankenhuysen 01a] M. L. Lapidus and M. van Frankenhuysen. "A Prime Orbit Theorem for SelfSimilar Flows and Diophantine Approximation." Contemporary Mathematics 290 (2001), 113-138.

[Lapidus and van Frankenhuysen 01b] M. L. Lapidus and M. van Frankenhuysen. "Complex Dimensions of SelfSimilar Fractal Strings and Diophantine Approximation." MSRI Preprint No. 2001-040, Berkeley, CA: Mathematical Sciences Research Institute (MSRI), 2001.

[Lenstra et al. 82] A. K. Lenstra, H. W. Lenstra, Jr., and L. Lovász. "Factoring Polynomials with Rational Coefficients." Math. Ann. 261 (1982), 515-534.

[Mandelbrot 83] B. B. Mandelbrot. The Fractal Geometry of Nature. New York: Freeman, 1983.

[Ostrowski 22] A. Ostrowski. "Bemerkungen zur Theorie der Diophantischen Approximationen." Abh. Math. Sem. Hamburg Univ. 1 (1922), 77-98.

[Parry and Pollicott 83] W. Parry and M. Pollicott. "An Analogue of the Prime Number Theorem and Closed Orbits of Axiom A Flows." Annals of Math. 118 (1983), 573-591.

[Parry and Pollicott 90] W. Parry and M. Pollicott. "Zeta Functions and the Periodic Orbit Structure of Hyperbolic Dynamics." In Astérisque, pp. 187-188, Paris: Soc. Math. France, 1990.
[Rössner and Schnorr] C. Rössner and C. P. Schnorr. "An Optimal, Stable Continued Fraction Algorithm for Arbitrary Dimension." In Proceedings of IPCO V (Conference on Integer Programming and Combinatorial Optimization, Vancouver, June 3-5, 1996), Lecture Notes in Computer Science 1084, pp. 31-43. Berlin: SpringerVerlag, 1996.

[Schmidt 80] W. M. Schmidt. Diophantine Approximation, Lecture Notes in Mathematics, 785, Berlin: SpringerVerlag, 1980.

[Strichartz 90] R. S. Strichartz. "Fourier Asymptotics of Fractal Measures." J. Functional Anal. 89 (1990), 154187.

[Strichartz 93] R. S. Strichartz. "Self-Similar Measures and their Fourier Transforms, I." Indiana Univ. Math. J. 39 (1990), 797-817; II, Trans. Amer. Math. Soc. 336 (1993), 335-361; III, Indiana Univ. Math. J. 42 (1993), 367-411.

[van Frankenhuysen 03] M. van Frankenhuysen. Available from World Wide Web (http://www.math. rutgers.edu/ ${ }^{\sim}$ machiel/programs/), 2003.

[Watson 95] G. N. Watson. A Treatise on the Theory of Bessel Functions, Second edition, Cambridge Mathematical Library. Cambridge, UK: Cambridge Univ. Press, 1995.

[Whittaker and Watson 96] E. T. Whittaker and G. N. Watson. A Course of Modern Analysis, Fourth edition, Cambridge Mathematical Library. Cambridge, UK: Cambridge Univ. Press, 1996.

Michel L. Lapidus, Department of Mathematics, University of California, Sproul Hall, Riverside, California 92521-0135 (lapidus@math.ucr.edu)

Machiel van Frankenhuysen, Department of Mathematics, Utah Valley State College, Orem, Utah 84058-5999 (vanframa@uvsc.edu)

Received June 29, 2001; accepted in revised form February 13, 2003. 